\title{
One-dimensional bargaining with unanimity rule
}

Citation for published version (APA):

Predtetchinski, A. (2007). One-dimensional bargaining with unanimity rule. METEOR, Maastricht University School of Business and Economics. METEOR Research Memorandum No. 011 https://doi.org/10.26481/umamet.2007011

Document status and date:

Published: 01/01/2007

DOI:

10.26481/umamet.2007011

Document Version:

Publisher's PDF, also known as Version of record

\section{Please check the document version of this publication:}

- A submitted manuscript is the version of the article upon submission and before peer-review. There can be important differences between the submitted version and the official published version of record.

People interested in the research are advised to contact the author for the final version of the publication, or visit the DOI to the publisher's website.

- The final author version and the galley proof are versions of the publication after peer review.

- The final published version features the final layout of the paper including the volume, issue and page numbers.

Link to publication

\footnotetext{
General rights rights.

- You may freely distribute the URL identifying the publication in the public portal. please follow below link for the End User Agreement:

www.umlib.nl/taverne-license

Take down policy

If you believe that this document breaches copyright please contact us at:

repository@maastrichtuniversity.nl

providing details and we will investigate your claim.
}

Copyright and moral rights for the publications made accessible in the public portal are retained by the authors and/or other copyright owners and it is a condition of accessing publications that users recognise and abide by the legal requirements associated with these

- Users may download and print one copy of any publication from the public portal for the purpose of private study or research.

- You may not further distribute the material or use it for any profit-making activity or commercial gain

If the publication is distributed under the terms of Article $25 \mathrm{fa}$ of the Dutch Copyright Act, indicated by the "Taverne" license above, 
Arkadi Predtetchinski

One-dimensional bargaining with unanimity rule

$\mathrm{RM} / 07 / 011$

JEL code: C78

\section{METEबrR}

Maastricht research school of Economics of TEchnology and ORganizations

Universiteit Maastricht

Faculty of Economics and Business Administration P.O. Box 616

NL - 6200 MD Maastricht

phone : ++31433883830

fax : ++31433884873 



\title{
One-dimensional bargaining with unanimity rule
}

\author{
Arkadi Predtetchinski*
}

March 27, 2007

\begin{abstract}
The paper examines bargaining over a one-dimensional set of social states, with a unanimity acceptance rule. We consider a class of $\delta$-equilibria, i.e. subgame perfect equilibria in stationary strategies that are free of coordination failures in the response stage. We show that along any sequence of $\delta$-equilibria, as $\delta$ converges to one, the proposal of each player converges to the same limit. The limit, called the bargaining outcome, is uniquely determined by the set of players, the recognition probabilities, and the utility functions, and it is independent of the choice of the sequence. We characterize the bargaining outcome as a unique solution of a characteristic equation.
\end{abstract}

JEL classification code: C78

Keywords: Bargaining, subgame perfect equilibrium, unanimity rule.

*I am most thankful to Jean-Jacques Herings to whom this work is dedicated. I would also like thank all the participants of a special seminar in the Sobolev Institute of Mathematics (Novosibirsk, Russia). 


\section{Introduction}

This paper studies bargaining in a group of players over a one-dimensional set of alternatives or social outcomes. The alternatives may represent the level of taxation, the amount of the public good, or a location of a facility.

Bargaining proceeds as follows. First, nature chooses a player to make a proposal. Recognition probabilities (i.e. the distribution on the moves of nature) are time-invariant. The chosen player then puts forward a proposal that specifies one alternative. The players react sequentially, in an exogenously given order. Each player can accept or reject a proposal. If the players unanimously agree to a proposal, it is implemented and the procedure ends. Otherwise, a new time period begins, and a new proposer is chosen. Each time a new period begins, a discount factor $\delta$ applies.

The central question of the paper is, of course, what alternative or alternatives will be eventually chosen. The paper gives a surprisingly sharp answer to the question as it identifies a specific alternative that is a unique outcome, in a sense made precise below, of the bargaining procedure.

It is clear that the model of bargaining with the unanimity rule as described above is not equally applicable to all instances where negotiations take place. However, there are important real-life examples where the unanimity is indeed required. Thus, peace or disarmament talks are likely to achieve a viable agreement if it is approved upon by all parties.

It is well-known that bargaining procedures such as the one above typically have infinitely many subgame perfect equilibria, including equilibria where delay occurs. For that reason we shall restrict attention to a class of subgame perfect equilibria involving only stationary strategies. Stationarity means that an equilibrium proposal of any player does not depend on the history of play and that the reaction of any player to a proposal only depends on the proposal itself.

When there are finitely many players, in a subgame perfect equilibrium involving only stationary strategies there is no delay. However, with infinitely many players, stationarity alone does not guarantee immediate acceptance of equilibrium proposals and even a perpetual disagreement can be supported as a subgame perfect equilibrium in stationary strategies. Despite the fact that players respond to a proposal sequentially, coordination failures can occur when a proposal is rejected by infinitely many players. We shall only be concerned with subgame perfect equilibria in stationary strategies that are robust to coordination failures in the response stage, in the sense that, whenever a proposal is rejected, for at least one player rejection remains a best response even if all other players, by mistake, accept. In any such equilibrium, no delay ever occurs.

Even in this class of equilibria, the individual acceptance sets are indeterminate. I shall further narrow down the class of strategies by looking at $\delta$-equilibria that pin down a specific functional form for the individual acceptance sets. Restricting attention to $\delta-$ equilibria is without loss of generality, however: in terms of equilibrium proposals and equilibrium utilities, the class of $\delta$-equilibria effectively represents all subgame perfect equilibria in stationary strategies that are robust to coordination failures in the response 
stage.

The concept of $\delta$-equilibrium as a solution concept for a social choice problem suffers from two obvious disadvantages. One disadvantage is that a given $\delta$-equilibrium does not unambiguously pin down a specific alternative, because different players make different proposals in equilibrium. Furthermore, it depends on the discount factor $\delta$. This motivates us to consider the asymptotic behavior of $\delta$-equilibria as $\delta$ converges to one.

We prove that along any sequence of $\delta$-equilibria, as the discount factor $\delta$ converges to one, the equilibrium proposals of all players converge to the same limit, and the social acceptance set collapses to a point. This point, called the bargaining outcome, is independent of the choice of the sequence and is uniquely determined by the set of players, recognition probabilities and the utility functions. The main result of the paper is a characterization of the bargaining outcome as the unique solution of a characteristic equation. The characterization is particularly simple in the special case where the player's utility of an alternative $x$ is a linear function of the distance of that player's ideal point from $x$. In this case, the bargaining outcome is the unique zero of the function $g$ that assigns to each alternative $x$ the mass of players with the ideal points in the interval $(x, 1]$.

In the special case of the model with two players, the bargaining outcome as defined above coincides with the asymmetric Nash bargaining solution, with weights equal to the respective recognition probabilities. In this case the characteristic equation is a first-order condition for the maximization of the asymmetric Nash product.

This paper is closely related to the work Cardona and Ponsati [2]. Cardona and Ponsati [2] study bargaining over a one-dimensional set of social outcomes, with a deterministic recognition rule where a passing of a proposal requires an approval of at least $q$ players. The authors prove stationary equilibrium to be asymptotically unique: for a given quota $q$, as the discount factor converges to one, the equilibrium proposals of all players approach the same limit, the limit being independent of the recognition sequence. While Cardona and Ponsati [2] prove asymptotic uniqueness of stationary equilibria, they do not provide an explicit computation of the limit. Our results are therefore complementary. We contribute to the insights in [2] by showing that the bargaining outcome can be characterized as a unique zero of a characteristic equation, but our results apply only to a game of bargaining with random recognition and the unanimity acceptance rule.

The setup of this paper is close that in Banks and Duggan [1] and Cho and Duggan [3]. Banks and Duggan [1] consider bargaining over a set of social outcomes that is an arbitrary compact convex subset of an Euclidean space. The bargaining protocol examined in Banks and Duggan [1] has time-invariant recognition probabilities and a general voting rule. A voting rule is represented by a family of decisive coalitions, and the approval of a proposal by any of these coalitions is sufficient for the passing of a proposal. Unanimity rule considered in this paper is a voting rule where the entire player set is the only decisive coalition. Banks and Duggan [1] prove existence of stationary equilibrium and examine the equilibrium set in the case of perfectly patient players (i.e. when the discount factor equals one). The setting in Cho and Duggan [3] is similar to that in Banks and Duggan [1], but the paper focuses on the case of a one-dimensional set of alternatives.

The bargaining protocol studied in this paper is the same as the bargaining protocol 
in Banks and Duggan [1] and Cho and Duggan [3] with the unanimity acceptance rule. In particular, the class of $\delta$-equilibria coincides with a class of stationary equilibria as defined in Cho and Duggan [3], under the unanimity rule.

This work builds on a contribution of Herings and Predtetchinski [4]. Herings and Predtetchinski [4] study a bargaining protocol where the identity of a proposer follows a Markov process, and player's utility of an alternative $x$ is a linear function of the distance of that player's ideal point from $x$. The setup of this paper is much more general with respect to the utility functions: it is only assumed that each utility function is continuous, concave and has a single peak (ideal point). On the other hand, we do not treat a general Markov recognition rule, but restrict attention to the case of time-invariant recognition probabilities.

As we have already mentioned, when there are only two players, the bargaining outcome in a model of one-dimensional coincides with the asymmetric Nash bargaining solution. With more than two players, the bargaining outcome does not in general maximize the (asymmetric) Nash product. The model of one-dimensional bargaining therefore provides a complementary set of the results to those in Miyakawa [5], where the asymmetric Nash bargaining solution for an $n$-person bargaining problem is obtained as a limit of stationary equilibria in a game of bargaining with time-invariant recognition probabilities, as the probability of the breakdown of negotiations converges to zero.

The rest of the paper is as follows. The next section presents the most important ideas and definitions including the definition of stationary strategy, a definition of $\delta$-equilibrium, and of bargaining outcome. Section 3 illustrates the key insights by means of an example. We consider a world where player's utility of an alternative $x$ depends linearly on the distance between the player's location and $x$. For this special case we prove that the bargaining outcome is the unique zero of the function $g$ that maps each alternative $x$ into the mass of players in the interval $(0, x]$.

Section 4 proves existence of $\delta$-equilibrium. Banks and Duggan [1] prove the existence of no-delay stationary equilibria, but they assume finitely many players. We extend the existence result to environments with an arbitrary player set, under appropriate continuity assumptions.

In Section 5 the set of 1-equilibria is analyzed. It is demonstrated that in each 1equilibrium the social acceptance set is a singleton. We also show that, given a weakly Pareto-efficient alternative, there exists a 1-equilibrium where each player proposes $x$. Furthermore, it is proved that along any sequence of $\delta$-equilibria as $\delta$ converges to one, the social acceptance set collapses to a point.

Section 6 introduces the characteristic function and the main result of the paper. The next section is devoted to the proof of the main result.

Section 8 proves that each $\delta$-equilibrium is a subgame perfect equilibrium. If the number of players is finite, for a given stationary subgame perfect equilibrium there exists a $\delta$-equilibrium with the same proposals and equilibrium utilities. More generally, if a subgame perfect equilibrium in stationary strategies is robust to coordination failures in the response stage, then there exists a $\delta$-equilibrium having the same proposals and equilibrium utilities. In this sense, $\delta$-equilibria effectively represent all subgame perfect equilibria in 
stationary strategies that are robust to coordination failures in the response stage.

\section{The components of the model}

\subsection{A description of the world and a game of bargaining}

We study a world $\omega$ described by the following variables: $X, N, \mathscr{A}, \mu$, and $u_{\bullet}$. The symbol $X$ denotes the unit interval $[0,1]$. This is a space of alternatives or social states the players must choose from. The set $N$ is a set of players that can be finite or infinite. The symbol $\mathscr{A}$ denotes a sigma-algebra of subsets of $N$, and $\mu$ is the probability measure. Thus the triple $(N, \mathscr{A}, \mu)$ is a probability space. The probability measure represents the distribution of types within the population. The symbol $u_{\bullet}$ denotes a collection of utility functions, one for each player. The utility function of player $t \in N$ is $u_{t}: X \rightarrow[0,1]$.

Without further mentioning we shall assume that the characteristics of the world satisfy the following assumption.

(A1) For each $t \in N$ the utility function $u_{t}: X \rightarrow[0,1]$ is concave, continuous, and it attains its unique maximum at point $\bar{x}_{t}$. The function $\bar{x}_{\bullet}: N \rightarrow X$ given by $t \mapsto \bar{x}_{t}$ is $\mathscr{A}$-measurable.

Given a discount factor $\delta \in[0,1]$ we define a game of bargaining $\Gamma(\delta)$ as follows. The game starts in period zero. Each period $\tau$ begins with nature randomly choosing a player from the set $N$ to make a proposal. A probability for a proposer to be a member of a set $S \in \mathscr{A}$ is $\mu(S)$. The chosen player proposes an alternative $x$ from $X$. All players (including the proposer) respond. We assume that the players resound sequentially, according to a total order $>$ on the player set $N$. The order $>$ is fixed throughout the game. Each responder can either accept or reject the current proposal. If the responders unanimously agree to the proposal, the game terminates and the proposal is implemented. Otherwise, period $\tau+1$ begins.

If alternative $x$ is agreed upon in period $\tau$, player $i$ receives a payoff of $\delta^{\tau} u_{i}(x)$. The payoff of perpetual disagreement to any player is zero.

It is well known that games of bargaining with more than two players typically have infinitely many subgame perfect equilibria, including equilibria with delay. In order to make a prediction about an outcome of the game, one would often restrict attention to a smaller class of equilibria, typically a class of subgame perfect equilibria involving only stationary strategies. This approach is also adopted in our paper. The exact definition of a profile of stationary strategies is as follows.

Definition 1 A joint strategy $\sigma$ is said to be stationary if there exist an $\mathscr{A}$-measurable function $x_{\bullet}: N \rightarrow X$ and a collection $A_{\bullet}$ of subsets $A_{t}$ of $X$, one for each $t \in N$, with $\cap A_{t}$ a Borel-measurable set such that (a) whenever player $t$ has to make a proposal, player $t$ proposes $x_{t}$, and (b) whenever player $t$ has to respond to a proposal $x$, player $t$ accepts if and only if $x \in A_{t}$. The set $A_{t}$ is called an individual acceptance set of player $t$ and $\cap A_{t}$ is called a social acceptance set. 


\subsection{A $\delta$-equilibrium and a bargaining solution}

We shall use the following solution concept for the game $\Gamma(\delta)$, called for brevity $\delta$ equilibrium.

Definition 2 Let $x_{\bullet}: N \rightarrow X$ be an $\mathscr{A}$-measurable function and $y_{\bullet}: N \rightarrow[0,1]$ be an arbitrary function. Let $A_{\bullet}$ be a collection of subsets $A_{t}$ of $X$ for $t \in N$. Let $A$ be a non-empty subset of $X$. The tuple $\left(x_{\bullet}, y_{\bullet}, A_{\bullet}, A\right)$ is said to be a $\delta$-equilibrium of the world $\omega$ if the following conditions are satisfied:

$$
\begin{gathered}
x_{t}=\arg \max _{x \in A} u_{t}(x) \text { for each } t \in N, \\
y_{t}=\int u_{t}\left(x_{i}\right) d \mu(i) \text { for each } t \in N, \\
A_{t}=\left\{x \in X \mid u_{t}(x) \geq \delta y_{t}\right\} \text { for each } t \in N, \\
A=\cap A_{t} .
\end{gathered}
$$

In a $\delta$-equilibrium no delay ever occurs, as all equilibrium proposals are unanimously accepted. As we show in Section 8 , if $\left(x_{\bullet}, y_{\bullet}, A_{\bullet}, A\right)$ is a $\delta$-equilibrium, then the joint stationary strategy $\left(x_{\bullet}, A_{\bullet}\right)$ is a subgame perfect equilibrium of the game $\Gamma(\delta)$. Conversely, if the player set $N$ is finite and the joint stationary strategy $\left(x_{\bullet}, A_{\bullet}\right)$ is a subgame perfect equilibrium, then the equilibrium proposal map $x_{\bullet}$ is a part of some $\delta$-equilibrium $\left(x_{\bullet}, \dot{y}_{\bullet}, \dot{A}_{\bullet}, \dot{A}\right)$. In particular, each $x_{t}$ is unanimously accepted. In general, if the joint stationary strategy $\left(x_{\bullet}, A_{\bullet}\right)$ is a subgame perfect equilibrium and is free of coordination failures in the response stage of the game (in the sense made precise in Section 8), then the equilibrium proposal map $x_{\bullet}$ is a part of some $\delta$-equilibrium $\left(x_{\bullet}, \dot{y}_{\bullet}, \dot{A}_{\bullet}, \dot{A}\right)$.

Since we assume utility functions to be concave, each individual acceptance set is an interval. We shall use the notation $\left[x_{t}^{-}, x_{t}^{+}\right]$to denote the individual acceptance set $A_{t}$ of player $t$. The social acceptance set is also a closed interval, denoted by $\left[x^{-}, x^{+}\right]$or $\left[x_{-}, x_{+}\right]$. Furthermore,

$$
x^{-}=\sup \left\{x_{t}^{-}\right\} \text {and } x^{+}=\inf \left\{x_{t}^{+}\right\},
$$

where the supremum and the infinum are taken over all $t \in N$. The equilibrium proposal $x_{t}$ of player $t$ is a point of $\left[x^{-}, x^{+}\right]$closest to $\bar{x}_{t}$, the ideal point of individual $t$. Thus

$$
x_{t}= \begin{cases}x^{-} & \text {if } \bar{x}_{t} \leq x^{-} \\ \bar{x}_{t} & \text { if } x^{-} \leq \bar{x}_{t} \leq x^{+} \\ x^{+} & \text {if } x^{+} \leq \bar{x}_{t}\end{cases}
$$

Notice that the function $x_{\bullet}$ thus defined is $\mathscr{A}$-measurable.

Definition 3 For each naturaln let $\left(x_{\bullet}^{n}, y_{\bullet}^{n}, A_{\bullet}^{n}, A^{n}\right)$ be a $\delta^{n}$-equilibrium of the world $\omega$ and let $x^{n}$ be a point in $A^{n}$. Suppose that the sequence $\delta^{n}$ converges to one and $x^{n}$ converges to $x$. Then the alternative $x$ is called a bargaining outcome of $\omega$. A collection of bargaining outcomes is called a bargaining solution. 
The main result of the paper states that the bargaining outcome is unique. In particular, along any sequence of $\delta$-equilibria, as $\delta$ converges to one, the social acceptance set collapses to a point, and in the limit all players make the same proposal. The next section illustrates this important insight by an example.

\subsection{General remarks}

For a subset $B$ of $\mathbb{R}$ we write $\operatorname{Int} B$ to denote the interior of $B$. In particular, $\operatorname{Int} X=(0,1)$.

Assumption (A1) implies that each function $u_{t}$ is positive on $\operatorname{Int} X$ and that it is not a constant on any non-degenerate interval $I \subset X$.

A concave function $f: X \rightarrow \mathbb{R}$ has left and right derivatives at each point $x$ of $\operatorname{Int} X$ denoted by $f^{\prime}(x-0)$ and $f^{\prime}(x+0)$. We shall often use the following fact: Given $x \in \operatorname{Int} X$ and $\dot{x} \in X$, the inequality $f(\dot{x})-f(x) \leq s(\dot{x}-x)$ holds for each $s \in\left[f^{\prime}(x-0), f^{\prime}(x+0)\right]$.

\section{An example}

This section builds on the results in Herings and Predtetchinski [4]. Consider a world $\lambda$ where $N \subset[0,1]$ is a finite or infinite set of players containing 0 and 1 . We assume that $\mathscr{A}$ is a sigma-algebra of Borel subsets of $N$. The utility function of player $t \in N$ is $u_{t}(x)=$ $1-|x-t|$. Define a function $g:[0,1] \rightarrow[0,1]$ by letting $g(x)=\mu(\{i \in N \mid x<i\})$. Then $1-g$ is the cumulative distribution function on $X$ induced by the probability measure $\mu$. The function $g$ is non-decreasing and it is continuous if $\mu$ is non-atomic, i.e. if $\mu(\{t\})=0$ for each $t \in N$. We need the following definition.

Definition 4 Point $x \in X$ is a generalized fixed point of the function $f: X \rightarrow X$ if there are sequences $x_{-}^{n}$ and $x_{+}^{n}$ of points in $X$ converging to $x$ such that $\lim f\left(x_{-}^{n}\right) \leq x \leq$ $\lim f\left(x_{+}^{n}\right)$.

It is clear that any fixed point $x$ of $f$ is also a generalized fixed point of $f$ (take $x_{-}^{n}=x$ and $x_{+}^{n}=x$ ). Conversely, if $f$ is continuous, then any generalized fixed point of $f$ is also its fixed point. If $f$ is a non-increasing function, then it has exactly one generalized fixed point.

\section{Proposition 1}

(i) For each $\delta \in[0,1)$ the world $\lambda$ has a unique $\delta$-equilibrium. The equilibrium proposals are given by Figure 1 below, where $\left[x^{-}, x^{+}\right]$is a social acceptance set.

(ii) Let $N=[0,1]$ and suppose that $\mu(B)=\mu(1-B)$ for each Borel-measurable set $B \subset N$, where $1-B=\{1-t \mid t \in B\}$. Then $x^{-}=\delta / 2$ and $x^{+}=1-\delta / 2$, and the unique bargaining outcome of the world $\lambda$ is $1 / 2$.

(iii) The world $\lambda$ has a unique bargaining outcome being the generalized fixed point of the function $g$. 


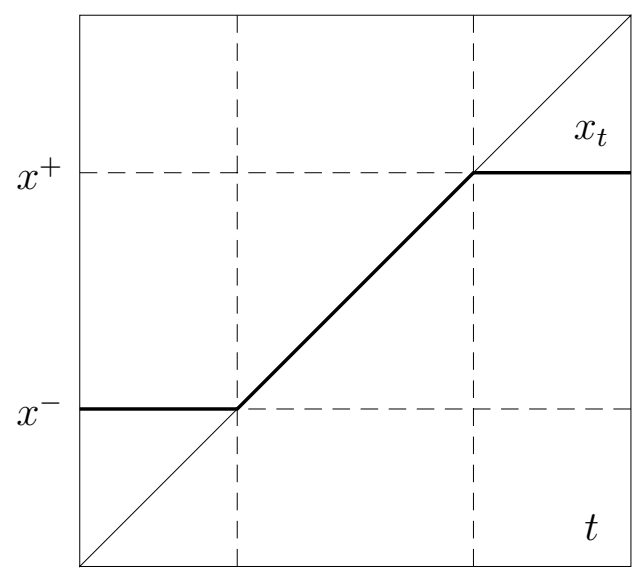

Figure 1: Equilibrium proposal $x_{t}$ in the example of Section 3.

Proof. Let $\left(x_{\bullet}, y_{\bullet}, A_{\bullet}, A\right)$ be a $\delta$-equilibrium of $\lambda$ and let $A_{t}=\left[x_{t}^{-}, x_{t}^{+}\right]$and $A=\left[x^{-}, x^{+}\right]$. First we show that the triple $\left(x_{\bullet}, x^{-}, x^{+}\right)$satisfies the following equations:

$$
\begin{gathered}
x_{t}= \begin{cases}x^{-} & \text {if } t \leq x^{-} \\
t & \text { if } x^{-} \leq t \leq x^{+} \\
x^{+} & \text {if } x^{+} \leq t,\end{cases} \\
x^{-}=\delta E\left(x_{\bullet}\right) \text { and } x^{+}=1-\delta+\delta E\left(x_{\bullet}\right) .
\end{gathered}
$$

The proposal $x_{t}$ of player $t$ is the point of $\left[x^{-}, x^{+}\right]$which is closest to $t$, as is illustrated in Figure 1 above, whence Equation 1. To derive Equation 2, we compute:

$$
\begin{gathered}
y_{t}=1-\int\left|x_{i}-t\right| d \mu(i), \\
x_{t}^{-}=\left(t-\left[1-\delta y_{t}\right]\right) \vee 0 \text { and } x_{t}^{+}=\left(t+\left[1-\delta y_{t}\right]\right) \wedge 1 .
\end{gathered}
$$

Now one can see that $x_{\bullet}^{-}: N \rightarrow X$ and $x_{\bullet}^{+}: N \rightarrow X$ are non-decreasing functions. It follows that $x^{-}=x_{1}^{-}$and $x^{+}=x_{0}^{+}$. Because $u_{1}(x)=x$, we have $y_{1}=E\left(x_{\bullet}\right)$ and therefore $x_{1}^{-}=\delta E\left(x_{\bullet}\right)$. Because $u_{0}(x)=1-x$, we have $y_{0}=1-E\left(x_{\bullet}\right)$ and therefore $x_{0}^{+}=1-\delta+\delta E\left(x_{\bullet}\right)$. Equation 2 follows.

Existence of $\delta$-equilibrium will be proven more generally in Section 4 . We prove that for each $\delta \in[0,1)$ the world $\lambda$ has at most one $\delta$-equilibrium by showing that the system 1-2 has at most one solution. Suppose the triples $\left(x_{\bullet}, x^{-}, x^{+}\right)$and $\left(\dot{x}_{\bullet}, \dot{x}^{-}, \dot{x}^{+}\right)$both satisfy Equations 1-2. Let $\left|\dot{x}_{\bullet}-x_{\bullet}\right|=\sup \left|\dot{x}_{t}-x_{t}\right|$. Then $\left|\dot{x}^{-}-x^{-}\right|=\left|\dot{x}^{+}-x^{+}\right|=\delta\left|E\left(\dot{x}_{\bullet}-x_{\bullet}\right)\right| \leq$ $\delta\left|\dot{x}_{\bullet}-x_{\bullet}\right|$. On the other hand, as we show in the proof of Proposition $3,\left|\dot{x}_{\bullet}-x_{\bullet}\right| \leq$ $\left|x^{-}-\dot{x}^{-}\right| \vee\left|x^{+}-\dot{x}^{+}\right|$. It follows that $\left(x_{\bullet}, x^{-}, x^{+}\right)=\left(\dot{x}_{\bullet}, \dot{x}^{-}, \dot{x}^{+}\right)$, as desired.

To prove claim (ii) let $x^{-}=\delta / 2$ and $x^{+}=1-\delta / 2$ and let $x_{\bullet}$ be as in Figure 1 . We show that $E\left(x_{\bullet}\right)=1 / 2$. It is then clear that the triple $\left(x_{\bullet}, x^{-}, x^{+}\right)$satisfies the system $1-2$. Since the system admits but one solution, $x_{\bullet}$ must be the equilibrium proposal map and $\left[x^{-}, x^{+}\right]$the social acceptance set in a $\delta$-equilibrium of $\lambda$. 
Since $x^{+}+x^{-}=1$, we have $x_{1-t}+x_{t}=1$ for all $t \in N$. Let $\left\{s_{\bullet}^{n}\right\}$ be a sequence of simple functions converging pointwise to $x_{\bullet}$. Define a function $\dot{s}_{\bullet}^{n}$ by the equation $\dot{s}_{t}^{n}=1 / 2\left(1+s_{t}^{n}-s_{1-t}^{n}\right)$ for all $t \in N$. The function $\dot{s}_{\bullet}^{n}$ is a simple function and the sequence $\left\{\dot{s}_{\bullet}^{n}\right\}$ converges pointwise to $x_{\bullet}$. Thus $E\left(\dot{s}_{\bullet}^{n}\right)$ converges to $E\left(x_{\bullet}\right)$.

It remains to show that $E\left(\dot{s}_{\bullet}^{n}\right)=1 / 2$. Suppose $s_{\bullet}^{n}=\sum a_{k} 1_{B_{k}}$, where $B_{k}$ are Borelmeasurable subsets of $N$. Then $\dot{s}_{\bullet}^{n}=1 / 2\left(1+\sum a_{k} 1_{B_{k}}-\sum a_{k} 1_{1-B_{k}}\right)$. Therefore, $E\left(\dot{s}_{\bullet}^{n}\right)=$ $1 / 2\left(1+\sum a_{k} \mu\left(B_{k}\right)-\sum a_{k} \mu\left(1-B_{k}\right)\right)=1 / 2$, because $\mu\left(B_{k}\right)=\mu\left(1-B_{k}\right)$ for all $k$.

We prove claim (iii). As before, let $\left(x_{\bullet}, y_{\bullet}, A_{\bullet}, A\right)$ be a $\delta$-equilibrium of $\lambda$ with $A=$ $\left[x^{-}, x^{+}\right]$. We estimate the expected equilibrium proposal from from below and from above, as follows. Define maps $z_{\bullet}^{-}: N \rightarrow X$ and $z_{\bullet}^{+}: N \rightarrow X$ by the following equations:

$$
z_{t}^{-}=\left\{\begin{array}{ll}
x^{-} & \text {if } t \in\left[0, x^{+}\right] \\
x^{+} & \text {otherwise }
\end{array} \quad \text { and } \quad z_{t}^{+}= \begin{cases}x^{-} & \text {if } t \in\left[0, x^{-}\right] \\
x^{+} & \text {otherwise }\end{cases}\right.
$$

Both maps are measurable and $z_{t}^{-} \leq x_{t} \leq z_{t}^{+}$for all $t \in N$. Therefore,

$$
\begin{gathered}
E\left(z_{\bullet}^{-}\right) \leq E\left(x_{\bullet}\right) \leq E\left(z_{\bullet}^{+}\right), \text {where } \\
E\left(z_{\bullet}^{-}\right)=\left(1-g\left(x^{+}\right)\right) x^{-}+g\left(x^{+}\right) x^{+}=(1-\delta) g\left(x^{+}\right)+\delta E\left(x_{\bullet}\right), \\
E\left(z_{\bullet}^{+}\right)=\left(1-g\left(x^{-}\right)\right) x^{-}+g\left(x^{-}\right) x^{+}=(1-\delta) g\left(x^{-}\right)+\delta E\left(x_{\bullet}\right) .
\end{gathered}
$$

Rewriting the above system yields

$$
g\left(x^{+}\right) \leq E\left(x_{\bullet}\right) \leq g\left(x^{-}\right) .
$$

Let $\left(x_{\bullet}^{n}, y_{\bullet}^{n}, A_{\bullet}^{n}, A^{n}\right)$ be a $\delta^{n}$-equilibrium of $\lambda$ with $A=\left[x_{-}^{n}, x_{+}^{n}\right]$. Suppose $\delta^{n}$ converges to one. Without loss of generality assume that both sequences $x_{-}^{n}$ and $x_{+}^{n}$ converge. Because $x_{+}^{n}-x_{-}^{n}=1-\delta^{n}$, the sequences $x_{-}^{n}$ and $x_{+}^{n}$ converge to the same limit, say a point $x$. The point $x$ is a bargaining outcome of $\lambda$. As $x_{-}^{n} \leq x_{t}^{n} \leq x_{+}^{n}$, the equilibrium proposal $x_{t}^{n}$ of each player $t$ also converges to $x$, and so does the expected value $E\left(x_{\bullet}^{n}\right)$. We know that $g\left(x_{+}^{n}\right) \leq E\left(x_{\bullet}^{n}\right) \leq g\left(x_{-}^{n}\right)$ for each $n$. Taking the limit, we find that $x$ is a generalized fixed point of $g$, as desired.

\section{Existence of $\delta$-equilibrium}

The results of this section rely on the following additional assumptions.

(A2) The inequality $\inf \left\{u_{t}\left(\bar{x}_{t}\right)\right\}>0$ holds.

(A3) The family of functions $u_{\bullet}$ is jointly continuous: Given an $x \in X$ and an $\epsilon>0$ there exists a $\varepsilon=\varepsilon(x, \epsilon)$ such that $\left|u_{t}(x)-u_{t}(\dot{x})\right|<\epsilon$ for all $t \in N$ whenever $|x-\dot{x}|<\varepsilon$. 
If the player set $N$ is finite, assumptions (A2) and (A3) are automatically satisfied. Indeed, assumption (A1) implies that $u_{t}\left(\bar{x}_{t}\right)>0$ for all $t \in N$, for otherwise the function $u_{t}$ would have been identically zero. Also, any finite family of continuous functions is jointly continuous.

In the general case, assumption (A1) implies that the family $u_{\bullet}$ is jointly continuous at any point $x$ in the interior of $X$, so that joint discontinuity can occur only at points 0 and 1. As an example of a family of utility functions that violates (A3) consider

$$
u_{t}(x)= \begin{cases}x / t, & \text { if } x \leq t \\ (1-x) /(1-t), & \text { if } x \geq t\end{cases}
$$

where the player set is $N=(0,1)$. Then the family $u_{\bullet}$ satisfies assumptions (A1) and (A2) but it is not uniformly continuous at either point of the boundary of $X$.

Consider also the following assumption.

(A4) The family of functions $u_{\bullet}$ is jointly uniformly continuous: Given an $\epsilon>0$ there exists a $\varepsilon=\varepsilon(\epsilon)$ such that $\left|u_{t}(x)-u_{t}(\dot{x})\right|<\epsilon$ for all $t \in N$ whenever $|x-\dot{x}|<\varepsilon$.

Assumption (A4) differs from (A3) in that $\varepsilon$ depends only on $\epsilon$ but not on $x$, whereas in (A3) it can depend on both. In fact, the two conditions are equivalent. To see this, let $F$ denote the set of all functions $N \rightarrow X$ endowed with a usual sup-norm: $\left|x_{\bullet}-\dot{x}_{\bullet}\right|=$ $\sup \left|x_{t}-\dot{x}_{t}\right|$ for $x_{\bullet}$ and $\dot{x}_{\bullet}$ in $F$. Let $u_{\bullet}$ denote a map $X \rightarrow F$ given by $x \mapsto u_{\bullet}(x)$. Then assumption (A3) can be equivalently stated as saying that the map $u_{\bullet}$ is continuous. Assumption (A4), on the other hand, is equivalent to a requirement that the map $u_{\bullet}$ be uniformly continuous. As $X$ is a compact space, any map from $X$ to a metric space is uniformly continuous if and only if it is continuous. Thus assumption (A3) can be replaced with (A4) without loss of generality.

Theorem 1 Let $\delta \in[0,1)$. Suppose $\omega$ satisfies the assumptions (A1), (A2) and (A3). Then $\omega$ has a $\delta$-equilibrium.

To prove Theorem 1 we characterize the $\delta$-equilibrium as a fixed point of a continuous map.

Let $F$ denote the set of all functions $N \rightarrow X$ endowed with a sup-norm. Let $D$ be a collection of all closed intervals $[a, b]$ in $X$. We shall identify $D$ with a subspace $\{(a, b) \in X \times X \mid a \leq b\}$ of $X \times X$. Let $M$ denote the set of all $\mathscr{A}$-measurable functions $x_{\bullet}: N \rightarrow X$. Let $Y$ be a set of maps $y_{\bullet}: N \rightarrow X$ such that there exists an $x \in X$ with $y_{t} \leq u_{t}(x)$ for all $t \in N$. The sets $M$ and $Y$ are considered subspaces of $F$. Let $S$ be a set of all families of closed intervals $A_{\bullet}$ in $X$ such that $\cap A_{t}$ is non-empty. Alternatively, one can think of $S$ as a set of pairs $\left(x_{\bullet}^{-}, x_{\bullet}^{+}\right)$of maps $x_{\bullet}^{-}: N \rightarrow X$ and $x_{\bullet}^{+}: N \rightarrow X$, such that $x_{t}^{-} \leq x \leq x_{t}^{+}$for all $t \in N$ for some $x \in X$. Then $S$ can be considered a subspace of $F \times F$.

Define $f$ to be a composite map

$$
D \stackrel{f_{1}}{\longrightarrow} M \stackrel{f_{2}}{\longrightarrow} Y \stackrel{f_{3}}{\longrightarrow} S \stackrel{f_{4}}{\longrightarrow} D, \text { where }
$$




$$
\begin{gathered}
f_{1}\left(x^{-}, x^{+}\right)_{t}= \begin{cases}x^{-} & \text {if } \bar{x}_{t} \leq x^{-} \\
\bar{x}_{t} & \text { if } x^{-} \leq \bar{x}_{t} \leq x^{+} \text {for each }\left(x^{-}, x^{+}\right) \in D, \\
x^{+} & \text {if } x^{+} \leq \bar{x}_{t}\end{cases} \\
f_{2}\left(x_{\bullet}\right)_{t}=\int_{i} u_{t}\left(x_{i}\right) d \mu(i) \text { for each } x_{\bullet} \in M, \\
f_{3}\left(y_{\bullet}\right)_{t}=\left\{x \in X \mid \delta y_{t} \leq u_{t}(x)\right\} \text { for each } y_{\bullet} \in Y, \\
f_{4}\left(A_{\bullet}\right)=\cap A_{t} \text { for each } A_{\bullet} \in S .
\end{gathered}
$$

The map $f_{4}$ can also be written as

$$
f_{4}\left(x_{\bullet}^{-}, x_{\bullet}^{+}\right)=\left(\sup \left\{x_{t}^{-}\right\}, \inf \left\{x_{t}^{+}\right\}\right) \text {for each }\left(x_{\bullet}^{-}, x_{\bullet}^{+}\right) \in S .
$$

It is obvious that an interval $\left[x^{-}, x^{+}\right]$is a social acceptance set in a $\delta$-bargaining outcome if and only if it is a fixed point of the map $f$. The map $f_{1}$ maps each interval $A$ to an equilibrium proposal map. The map $f_{2}$ transforms equilibrium proposals into a collection of expected utilities, whereas $f_{3}$ maps expected utilities to individual acceptance sets. Finally, $f_{4}$ maps individual acceptance sets to a social acceptance set.

It remains to show that the map $f$ is continuous. First we establish the following auxiliary proposition. Define

$$
m=\inf \left\{\left|u_{t}^{\prime}(x-0)\right| \mid \begin{array}{c}
(t, x) \in N \times \operatorname{Int} X \\
u_{t}(x) \leq \delta u_{t}\left(\bar{x}_{t}\right)
\end{array}\right\} .
$$

Proposition 2 If $\delta \in[0,1)$, then $m>0$.

Proof. Let $t \in N$ and $x \in \operatorname{Int} X$ be such that $u_{t}(x) \leq \delta u_{t}\left(\bar{x}_{t}\right)$. Then $u_{t}\left(\bar{x}_{t}\right)-u_{t}(x) \leq$ $u_{t}^{\prime}(x-0)\left(\bar{x}_{t}-x\right)$. We have thus the following chain of inequalities:

$$
\left|u_{t}^{\prime}(x-0)\right| \geq \frac{u_{t}\left(\bar{x}_{t}\right)-u_{t}(x)}{\left|\bar{x}_{t}-x\right|} \geq \frac{(1-\delta) u_{t}\left(\bar{x}_{t}\right)}{\left|\bar{x}_{t}-x\right|} \geq(1-\delta) u_{t}\left(\bar{x}_{t}\right) .
$$

It follows that

$$
m \geq(1-\delta) \inf \left\{u_{t}\left(\bar{x}_{t}\right)\right\}
$$

Assumption (A2) now implies that $m$ is positive.

Proposition 3 If $\delta \in[0,1)$, then the map $f$ is continuous.

Proof. The map $f_{1}$ is continuous. Let $A=\left[x^{-}, x^{+}\right]$and $\dot{A}=\left[\dot{x}^{-}, \dot{x}^{+}\right]$. Let $f_{1}(A)=x$. and $f_{1}(\dot{A})=\dot{x}_{\bullet}$. The continuity of $f_{1}$ follows from the inequality

$$
\left|x_{\bullet}-\dot{x}_{\bullet}\right| \leq\left|x^{-}-\dot{x}^{-}\right| \vee\left|x^{+}-\dot{x}^{+}\right| .
$$


To prove the inequality, fix a $t$ and let $\epsilon=\left|x^{-}-\dot{x}^{-}\right| \vee\left|x^{+}-\dot{x}^{+}\right|$. First we show that there is a point in $A$ at a distance of at most $\epsilon$ from $\dot{x}_{t}$. Similarly, there is a point in $\dot{A}$ at a distance of at most $\epsilon$ from $x_{t}$. To see this, write $x_{t}$ as a convex combination of $x^{-}$and $x^{+}$and $\dot{x}_{t}$ as a convex combination of $\dot{x}^{-}$and $\dot{x}^{+}: x_{t}=a x^{-}+(1-a) x^{+}$ and $\dot{x}_{t}=\dot{a} \dot{x}^{-}+(1-\dot{a}) \dot{x}^{+}$, and define the points $\dot{z}_{t} \in \dot{A}$ and $z_{t} \in A$ by the equations $\dot{z}_{t}=a \dot{x}^{-}+(1-a) \dot{x}^{+}$and $z_{t}=\dot{a} x^{-}+(1-\dot{a}) x^{+}$. Then

$$
\begin{aligned}
& \left|\dot{z}_{t}-x_{t}\right| \leq a\left|\dot{x}^{-}-x^{-}\right|+(1-a)\left|\dot{x}^{+}-x^{+}\right| \leq \epsilon \text { and } \\
& \left|z_{t}-\dot{x}_{t}\right| \leq \dot{a}\left|\dot{x}^{-}-x^{-}\right|+(1-\dot{a})\left|\dot{x}^{+}-x^{+}\right| \leq \epsilon .
\end{aligned}
$$

We also have the following obvious property: Let $x \in A$. If $x<x_{t}$, then $x_{t} \leq \bar{x}_{t}$ and if $x_{t}<x$, then $\bar{x}_{t} \leq x_{t}$. A similar property holds for $\dot{A}$.

Now we prove that $x_{t} \leq \dot{x}_{t}+\epsilon$. Suppose not. Then we have $z_{t} \leq \dot{x}_{t}+\epsilon<x_{t}$. Since $z_{t}$ is a point of $A$, this means that $x_{t} \leq \bar{x}_{t}$. On the other hand, we also have $\dot{x}_{t}<x_{t}-\epsilon \leq \dot{z}_{t}$. Since $\dot{z}_{t}$ is a point of $\dot{A}$, we have $\bar{x}_{t} \leq \dot{x}_{t}$. We arrive at a contradiction, because $\bar{x}_{t} \leq \dot{x}_{t}<x_{t}-\epsilon<x_{t} \leq \bar{x}_{t}$. The proof that $\dot{x}_{t} \leq x_{t}+\epsilon$ is similar.

The map $f_{2}$ is continuous. Let $f_{2}\left(x_{\bullet}\right)=y_{\bullet}$ and $f_{2}\left(\dot{x}_{\bullet}\right)=\dot{y}_{\bullet}$. Let $\epsilon$ and $\varepsilon$ be as in assumption (A4). Suppose $\left|x_{\bullet}-\dot{x}_{\bullet}\right|<\varepsilon$. Then $\left|u_{t}\left(x_{i}\right)-u_{t}\left(\dot{x}_{i}\right)\right|<\epsilon$ for all $t$ and $i$ in $N$. Therefore,

$$
\left|y_{t}-\dot{y}_{t}\right| \leq \int_{i}\left|u_{t}\left(x_{i}\right)-u_{t}\left(\dot{x}_{i}\right)\right| d \mu(i) \leq \epsilon .
$$

It follows that $\left|y_{\bullet}-\dot{y}_{\bullet}\right| \leq \epsilon$.

The map $f_{3}$ is continuous. Let $f_{3}\left(y_{\bullet}\right)=\left(x_{\bullet}^{-}, x_{\bullet}^{+}\right)$and $f_{3}\left(\dot{y}_{\bullet}\right)=\left(\dot{x}_{\bullet}^{-}, \dot{x}_{\bullet}^{+}\right)$. The continuity of $f_{3}$ is implied by the inequalities

$$
\left|x_{\bullet}^{-}-\dot{x}_{\bullet}^{-}\right| \leq \frac{\delta\left|y_{\bullet}-\dot{y}_{\bullet}\right|}{m} \text { and }\left|x_{\bullet}^{+}-\dot{x}_{\bullet}^{+}\right| \leq \frac{\delta\left|y_{\bullet}-\dot{y}_{\bullet}\right|}{m} .
$$

These inequalities are proven by showing that

$$
\left|x_{t}^{-}-\dot{x}_{t}^{-}\right| \leq \frac{\delta\left|y_{t}-\dot{y}_{t}\right|}{m} \text { and }\left|x_{t}^{+}-\dot{x}_{t}^{+}\right| \leq \frac{\delta\left|y_{t}-\dot{y}_{t}\right|}{m}
$$

for all $t \in N$. We prove the first set of inequalities. The proof of the second set of inequalities is analogous.

Recall that

$$
x_{t}^{-}=\inf \left\{x \in X \mid \delta y_{t} \leq u_{t}(x)\right\} \text { and } \dot{x}_{t}^{-}=\inf \left\{x \in X \mid \delta \dot{y}_{t} \leq u_{t}(x)\right\} .
$$

Thus, $\delta y_{t} \leq u_{t}\left(x_{t}^{-}\right)$and $\delta \dot{y}_{t} \leq u_{t}\left(\dot{x}_{t}^{-}\right)$. Without loss of generality, assume $\dot{x}_{t}^{-}<x_{t}^{-}$. In particular, $0<x_{t}^{-}$, implying the equality $\delta y_{t}=u_{t}\left(x_{t}^{-}\right)$. Notice that both points $\dot{x}_{t}^{-}$ and $x_{t}^{-}$lie in the interval $\left[0, \bar{x}_{t}\right)$, where $u_{t}$ is an increasing function. Thus the inequality $u_{t}\left(\dot{x}_{t}^{-}\right)<u_{t}\left(x_{t}^{-}\right)$holds. Also, the left derivative of $u_{t}$ at $x_{t}^{-}$is positive. Thus, we have the inequalities

$$
0<u_{t}^{\prime}\left(x_{t}^{-}-0\right)\left(x_{t}^{-}-\dot{x}_{t}^{-}\right) \leq u_{t}\left(x_{t}^{-}\right)-u_{t}\left(\dot{x}_{t}^{-}\right) \leq \delta\left(y_{t}-\dot{y}_{t}\right)
$$


They yield the inequalities

$$
0<x_{t}^{-}-\dot{x}_{t}^{-} \leq \frac{\delta\left(y_{t}-\dot{y}_{t}\right)}{u_{t}^{\prime}\left(x_{t}^{-}-0\right)}
$$

Since $y_{\bullet}$ is an element of $Y$, we have the inequality $y_{t} \leq u_{t}\left(\bar{x}_{t}\right)$. Therefore, $u_{t}\left(x_{t}^{-}\right)=$ $\delta y_{t} \leq \delta u_{t}\left(\bar{x}_{t}\right)$. It follows that $m \leq\left|u_{t}^{\prime}\left(x_{t}^{-}-0\right)\right|$. This gives the desired inequality

$$
0<x_{t}^{-}-\dot{x}_{t}^{-} \leq \frac{\delta\left(y_{t}-\dot{y}_{t}\right)}{m}
$$

The map $f_{4}$ is continuous. Let $f_{4}\left(x_{\bullet}^{-}, x_{\bullet}^{+}\right)=\left(x^{-}, x^{+}\right)$and $f_{4}\left(\dot{x}_{\bullet}^{-}, \dot{x}_{\bullet}^{+}\right)=\left(\dot{x}^{-}, \dot{x}^{+}\right)$. We have an obvious inequality $\left|x_{t}^{-}-\dot{x}_{t}^{-}\right| \leq\left|x_{\bullet}^{-}-\dot{x}_{\bullet}^{-}\right|$. Then $x_{t}^{-}-\left|x_{\bullet}^{-}-\dot{x}_{\bullet}^{-}\right| \leq \dot{x}_{t}^{-} \leq \dot{x}^{-}$ for all $t$, hence $x^{-}-\left|x_{\bullet}^{-}-\dot{x}_{\bullet}^{-}\right| \leq \dot{x}^{-}$. Also, $\dot{x}_{t}^{-}-\left|x_{\bullet}^{-}-\dot{x}_{\bullet}^{-}\right| \leq x_{t}^{-} \leq x^{-}$for all $t$, hence $\dot{x}^{-}-\left|x_{\bullet}^{-}-\dot{x}_{\bullet}^{-}\right| \leq x^{-}$. Thus, $\left|x^{-}-\dot{x}^{-}\right| \leq\left|x_{\bullet}^{-}-\dot{x}_{\bullet}^{-}\right|$. Similarly one derives an inequality $\left|x^{+}-\dot{x}^{+}\right| \leq\left|x_{\bullet}^{+}-\dot{x}_{\bullet}^{+}\right|$. Together they imply the continuity of $f_{4}$.

\section{The asymptotic behavior of $\delta$-equilibria}

This section is to devoted to Theorems 2 and 3 below. The first of these describes the set of $\delta$-equilibria when $\delta=1$ and shows that in each 1 -equilibrium the social acceptance set is a singleton. The second theorem shows that along any sequence of $\delta$-equilibria as $\delta$ converges to one, the social acceptance set collapses to a point.

An alternative $x$ is said to be weakly Pareto-efficient if there is no $\dot{x} \in X$ such that $u_{t}(\dot{x})>u_{t}(x)$ for all $t \in N$. Each alternative in the open interval $\left(\inf \left\{\bar{x}_{t}\right\}, \sup \left\{\bar{x}_{t}\right\}\right)$ is weakly Pareto-efficient. Furthermore, all alternatives in $\cup\left\{\bar{x}_{t}\right\}$ are obviously weakly Pareto-efficient. On the other hand, each weakly Pareto-efficient alternative lies in the closed interval $\left[\inf \left\{\bar{x}_{t}\right\}, \sup \left\{\bar{x}_{t}\right\}\right]$. If the player set is infinite it is easy to construct a family of the utility functions in such a way that the alternatives $\inf \left\{\bar{x}_{t}\right\}$ and $\sup \left\{\bar{x}_{t}\right\}$ are not weakly efficient.

Theorem 2 In each 1-equilibrium the social acceptance set is a singleton. Conversely, given a weakly Pareto-efficient alternative $x$, there exists a 1-equilibrium with a social acceptance set consisting of point $x$ alone.

As in the preceding section, we shall identify an interval $[a, b] \subset X$ with a point $(a, b) \in$ $X \times X$. Thus the collection of all closed intervals in $X$ can be seen as a metric space.

Theorem 3 For each natural number n let $A^{n}$ be a social acceptance set in a $\delta^{n}$-equilibrium. If the sequence $A^{n}$ converges to an interval $A$ and the sequence $\delta^{n}$ converges to 1 , then $A$ is a singleton.

The second part of Theorem 2 is easy to prove. Thus let $x$ be a weakly Pareto-efficient alternative. Define $\left(x_{\bullet}, y_{\bullet}, A_{\bullet}, A\right)$ by letting $x_{\bullet}$ be identically equal to $x, y_{t}=u_{t}(x)$ and 
$A_{t}=\left\{\dot{x} \in X \mid u_{t}(\dot{x}) \geq u_{t}(x)\right\}$ for all $t \in N$ and $A=\cap A_{t}$. We show that the set $A$ consists of point $x$ alone. It then follows that $\left(x_{\bullet}, y_{\bullet}, A_{\bullet}, A\right)$ is a 1 -equilibrium.

It is clear that $x$ is an element of $A$. Suppose $A$ contains an open interval $I$. For each point $\dot{x}$ in $I$ it must hold that $u_{t}(\dot{x})>u_{t}(x)$, for otherwise the function $u_{t}$ would be constant on $I$, contradicting assumption (A1). But then $x$ is not a weakly Pareto-efficient alternative, a contradiction. It follows that $A$ is a singleton.

To prove the rest of Theorem 2 and Theorem 3 it is convenient to consider an auxiliary concept of pseudo-equilibrium.

Definition 5 Let $x_{\bullet}: N \rightarrow X$ be an $\mathscr{A}$-measurable function and $y_{\bullet}: N \rightarrow[0,1]$ be an arbitrary function. Let $A_{\bullet}$ be a collection of subsets $A_{t}$ of $X$ for $t \in N$. Let $A$ be a non-empty subset of $X$. The tuple $\left(x_{\bullet}, y_{\bullet}, A_{\bullet}, A\right)$ is said to be a pseudo-equilibrium if it satisfies the first two conditions of Definition 2 and

$$
\begin{gathered}
A_{t}=\left\{x \in X \mid u_{t}(x) \geq y_{t}\right\} \text { for each } t \in N, \\
A \subset \cap A_{t} .
\end{gathered}
$$

It is clear that a 1-equilibrium is also a pseudo-equilibrium. Thus Proposition 4 below implies the first part of Theorem 2. Together Propositions 4 and 5 imply Theorem 3.

Proposition 4 If $\left(x_{\bullet}, y_{\bullet}, A_{\bullet}, A\right)$ is a pseudo-equilibrium, then $A$ is a singleton.

Proof. Let $\left(x_{\bullet}, y_{\bullet}, A_{\bullet}, A\right)$ be a pseudo-equilibrium. For each natural number $n$ define the set $A_{t}^{n}=\left\{x \in X \mid u_{t}(x) \geq y_{t}+1 / n\right\}$. Observe that $A_{t}^{n}$ is Borel-measurable being a closed subset of $X$. Consequently, the set $\left\{i \in N \mid x_{i} \in A_{t}^{n}\right\}$ is $\mathscr{A}$-measurable. Now,

$$
\begin{gathered}
y_{t}=\int_{\left\{i \in N \mid x_{i} \in A_{t}^{n}\right\}} u_{t}\left(x_{i}\right) d \mu(i)+\int_{\left\{i \in N \mid x_{i} \in A_{t}^{n}\right\}^{c}} u_{t}\left(x_{i}\right) d \mu(i) \geq \\
\geq\left(y_{t}+1 / n\right) \mu\left(\left\{i \in N \mid x_{i} \in A_{t}^{n}\right\}\right)+y_{t} \mu\left(\left\{i \in N \mid x_{i} \in A_{t}^{n}\right\}^{c}\right)= \\
=y_{t}+(1 / n) \mu\left(\left\{i \in N \mid x_{i} \in A_{t}^{n}\right\}\right) .
\end{gathered}
$$

Thus we must have $\mu\left(\left\{i \in N \mid x_{i} \in A_{t}^{n}\right\}\right)=0$. Define $B_{t}=\left\{x \in X \mid u_{t}(x)>y_{t}\right\}$. Clearly, $B_{t}=\cup_{n=1}^{\infty} A_{t}^{n}$. Therefore,

$$
\mu\left(\left\{i \in N \mid x_{i} \in B_{t}\right\}\right) \leq \sum_{n=1}^{\infty} \mu\left(\left\{i \in N \mid x_{i} \in A_{t}^{n}\right\}\right)=0 .
$$

We shall prove that there is a player $t \in N$ such that $u_{t}\left(x_{t}\right)=y_{t}$. If this is indeed the case, then the function $u_{t}$ is constant on the set $A$, because $u_{t}\left(x_{t}\right)$ is an upper bound for $u_{t}$ on the set $A$, while $y_{t}$ is a lower bound. Because $A$ is convex and $u_{t}$ is strictly quasi-concave, it would then follow that $A$ is singleton.

Suppose that $u_{t}\left(x_{t}\right)>y_{t}$ for all $t \in N$. Thus $x_{t} \in B_{t}$, so that the family of sets $\left\{B_{t}\right\}_{t \in N}$ is an open cover of the set $\cup_{t \in N}\left\{x_{t}\right\}$. Then there exists a countable subset $C \subseteq N$ such 
that the subfamily $\left\{B_{t}\right\}_{t \in C}$ covers $\cup_{t \in N}\left\{x_{t}\right\}$. This is a consequence of the fact that $X$ has a countable base; thus any open cover has a countable subcover.

It follows that

$$
N=\bigcup_{t \in C}\left\{i \in N \mid x_{i} \in B_{t}\right\}
$$

Therefore,

$$
\mu(N) \leq \sum_{t \in C} \mu\left(\left\{i \in N \mid x_{i} \in B_{t}\right\}\right)=0
$$

which is impossible because $\mu(N)=1$. The result follows.

Proposition 5 Let $\left(x_{\bullet}^{n}, y_{\bullet}^{n}, A_{\bullet}^{n}, A^{n}\right)$ be a sequence of $\delta^{n}$-equilibria. Suppose the sequence $A^{n}$ converges to an interval $A$ and the sequence $\delta^{n}$ converges to 1 . Then there exist maps $x_{\bullet}$ and $y_{\bullet}$ and a collection of sets $A_{\bullet}$ such that $\left(x_{\bullet}, y_{\bullet}, A_{\bullet}, A\right)$ is a pseudo-equilibrium.

Proof. Define $x_{\bullet}, y_{\bullet}$ and $A_{\bullet}$ by the following equations

$$
\begin{gathered}
x_{t}=\arg \max _{x \in A} u_{t}(x) \text { for each } t \in N, \\
y_{t}=\int u_{t}\left(x_{i}\right) d \mu(i) \text { for each } t \in N, \\
A_{t}=\left\{x \in X \mid u_{t}(x) \geq y_{t}\right\} \text { for each } t \in N .
\end{gathered}
$$

Let the map $f_{1}$ be as in Section 4. Recall that $f_{1}$ carries an interval $A$ into a map $x_{\bullet}$ where each $x_{t}$ is the point of $A$ closest to $\bar{x}_{t}$. Thus $x_{\bullet}^{n}=f_{1}\left(A^{n}\right)$ and $x_{\bullet}=f_{1}(A)$. As we have seen in the proof of Proposition 3, the map $f_{1}$ is continuous with respect to the topology of unform convergence. Therefore, the sequence $x_{\bullet}^{n}$ converges uniformly to $x_{\bullet}$.

Since each function $u_{t}$ is continuous, the sequence $u_{t}\left(x_{i}^{n}\right)$ converges to $u_{t}\left(x_{i}\right)$ for all $i \in$ $N$. Because the integral is continuous with respect to a topology of pointwise convergence, $y_{t}^{n}$ converges to $y_{t}$ for all $t \in N$. (We do not claim that the sequence $y_{\bullet}^{n}$ converges uniformly to $y_{\bullet}$. This is not true, unless we assume that the family $u_{\bullet}$ is jointly continuous).

Let $A^{n}=\left[x_{-}^{n}, x_{+}^{n}\right]$ and $A=\left[x^{-}, x^{+}\right]$. We know that $A^{n} \subset \cap A_{t}^{n}$. In particular, $u_{t}\left(x_{-}^{n}\right) \geq \delta^{n} y_{t}^{n}$ and $u_{t}\left(x_{+}^{n}\right) \geq \delta^{n} y_{t}^{n}$ for all $t \in N$. Taking the limits, we obtain the inequalities $u_{t}\left(x^{-}\right) \geq y_{t}$ and $u_{t}\left(x^{+}\right) \geq y_{t}$. This implies that $A \subset \cap A_{t}$, as desired.

\section{The characteristic function}

For each $x \in \operatorname{Int} X$ we define

$$
\begin{aligned}
& \varphi^{-}(x)=\inf \left\{\frac{u_{t}^{\prime}(x-0)}{u_{t}(x)}\right\}, \varphi^{+}(x)=\sup \left\{\frac{u_{t}^{\prime}(x+0)}{u_{t}(x)}\right\} \text { and } \\
& \xi(x)=\mu\left(\left\{i \in N \mid \bar{x}_{i}<x\right\}\right) \varphi^{-}(x)+\mu\left(\left\{i \in N \mid x<\bar{x}_{i}\right\}\right) \varphi^{+}(x) .
\end{aligned}
$$


Claim (a) of Proposition 9 implies that the numbers $\varphi^{-}(x), \varphi^{+}(x)$ and $\xi(x)$ are finite. The function $\xi$ is referred to as a characteristic function. It will be convenient to extend the characteristic function to $X$ by letting $\xi(0)=+\infty$ and $\xi(1)=-\infty$.

There is nothing particularly important about the use of left derivatives in the function $\varphi^{-}$and right derivatives in $\varphi^{+}$. As the reader can verify, all results remain true if one replaces a derivative $u_{t}^{\prime}(x-0)$ or $u_{t}^{\prime}(x+0)$ by an arbitrary element in the subgradient $\left[u_{t}^{\prime}(x+0), u_{t}^{\prime}(x-0)\right]$ of the function $u_{t}$.

Proposition 6 The function $\varphi^{+}$is positive and decreasing on the interval $\left(0, \sup \left\{\bar{x}_{i}\right\}\right)$. The function $\varphi^{-}$is negative and decreasing on the interval $\left(\inf \left\{\bar{x}_{i}\right\}, 1\right)$. The characteristic function $\xi$ is decreasing on $X$.

Proof. Let $\ell_{t}=\ln u_{t}$. The function is well-defined on $(0,1)$ because $u_{t}(x)>0$ for all $x \in(0,1)$. Then we can write

$$
\varphi^{-}(x)=\inf \left\{\ell_{t}^{\prime}(x-0)\right\} \text { and } \varphi^{+}(x)=\sup \left\{\ell_{t}^{\prime}(x+0)\right\} .
$$

Since $\ell_{t}$ is a concave function, both its left and right derivatives are non-increasing. It follows that both $\varphi^{-}$and $\varphi^{+}$are non-increasing functions.

We prove that $\varphi^{+}$is positive on the interval $\left(0, \sup \left\{\bar{x}_{i}\right\}\right)$. Given $0<x<\sup \left\{\bar{x}_{i}\right\}$, there exists a player $t$ such that $x<\bar{x}_{t}$. For this player $t$ it holds that $u_{t}^{\prime}(x+0)>0$, therefore also $\ell_{t}^{\prime}(x+0)>0$. It follows that $\varphi^{+}(x)>0$, as desired.

We prove that $\varphi^{+}$is decreasing on $\left(0, \sup \left\{\bar{x}_{i}\right\}\right)$. Suppose not. Then there exist $0<$ $a<b<\sup \left\{\bar{x}_{i}\right\}$ such that $\varphi^{+}(a) \leq \varphi^{+}(b)$. As we already know that the function $\varphi^{+}$is non-increasing, it has to be a constant (say, equal to $m$ ) on the interval $[a, b]$. Let $t^{q}$ be a sequence in $N$ such that $\ell_{t^{q}}^{\prime}(b+0) \rightarrow m$. Then for each $x \in[a, b]$ there are the inequalities

$$
m \geq \ell_{t^{q}}^{\prime}(a+0) \geq \ell_{t^{q}}^{\prime}(x+0) \geq \ell_{t^{q}}^{\prime}(b+0),
$$

where the right-hand side converges to $m$ as $q$ goes to infinity. The first of these inequalities follows from the fact that $m$ is the supremum of $\ell_{t}^{\prime}(a+0)$, while the other two are true because the right derivative of $\ell_{t}$ is a non-increasing function. It follows that $\ell_{t^{q}}^{\prime}(x+0) \rightarrow m$. Now we have the inequalities

$$
\ell_{t^{q}}^{\prime}(x+0)(x-a) \leq \ell_{t^{q}}(x)-\ell_{t^{q}}(a) \leq \ell_{t^{q}}^{\prime}(a+0)(x-a)
$$

where the left- and the right-hand sides converge to $m(x-a)$. Thus $\ell_{t^{q}}(x) \rightarrow \ell_{t^{q}}(a)+m(x-$ $a$ ). Since $x \in[a, b]$ is arbitrary, it means that the sequence $\left\{\ell_{t^{q}}\right\}$ of functions converges pointwise on $[a, b]$ to a linear function $c+m(\bullet-a)$. Therefore, the sequence $\left\{u_{t^{q}}\right\}$ of functions converges pointwise on $[a, b]$ to an exponential function $\exp (c) \exp (m(\bullet-a))$. But this is impossible, because each $u_{t^{q}}$ is a concave function, and the set of concave functions on a compact interval is closed in the topology of pointwise convergence. This contradiction establishes that $\varphi^{+}$is decreasing on the interval $\left(0, \sup \left\{\bar{x}_{i}\right\}\right)$.

The proof that $\varphi^{-}$is negative and decreasing on $\left(\inf \left\{\bar{x}_{i}\right\}, 1\right)$ is similar. 
Write $\mu\left(\bar{x}_{i}<x\right)$ to denote $\mu\left(\left\{i \in N \mid \bar{x}_{i}<x\right\}\right)$ and write $\mu\left(x<\bar{x}_{i}\right)$ to denote $\mu(\{i \in$ $\left.\left.N \mid x<\bar{x}_{i}\right\}\right)$. Consider the sets

$$
X_{-}=\left\{x \in \operatorname{Int} X \mid \mu\left(\bar{x}_{i}<x\right)>0\right\} \text { and } X_{+}=\left\{x \in \operatorname{Int} X \mid \mu\left(x<\bar{x}_{i}\right)>0\right\},
$$

and the sets $X_{-}^{c}=\operatorname{Int} X-X_{-}$and $X_{+}^{c}=\operatorname{Int} X-X_{+}$. It is clear that the following inclusions hold:

$$
X_{-} \subset\left(\inf \left\{\bar{x}_{i}\right\}, 1\right) \text { and } X_{+} \subset\left(0, \sup \left\{\bar{x}_{i}\right\}\right) .
$$

Furthermore, the set $X_{-}^{c} \cap X_{+}^{c}$ does not contain a non-degenerate interval. For suppose that $[a, b] \subset X_{-}^{c} \cap X_{+}^{c}$ where $a<b$. Then $\mu(N) \leq \mu\left(\bar{x}_{i}<b\right)+\mu\left(a<\bar{x}_{i}\right)=0$, a contradiction.

Consider the function $\xi^{+}(\bullet)=\mu\left(\bullet<\bar{x}_{i}\right) \varphi^{+}(\bullet)$. The function $\mu\left(\bullet<\bar{x}_{i}\right)$ is identically zero on $X_{+}^{c}$. On the set $X_{+}$the function $\mu\left(\bullet<\bar{x}_{i}\right)$ is positive and non-increasing while the function $\varphi^{+}$is positive and decreasing. It follows that the function $\xi^{+}$is identically zero on $X_{+}^{c}$ and is positive and decreasing on $X_{+}$. Similarly, consider the function $\xi^{-}(\bullet)=\mu\left(\bar{x}_{i}<\right.$ $\bullet) \varphi^{-}(\bullet)$. The function $\mu\left(\bar{x}_{i}<\bullet\right)$ is identically zero on $X_{-}^{c}$. Furthermore, $\mu\left(\bar{x}_{i}<\bullet\right)$ is positive and non-decreasing on $X_{-}$, while $\varphi^{-}$is negative and decreasing on $X_{-}$. It follows that $\xi^{-}$identically zero on $X_{-}^{c}$ and is negative and decreasing on $X_{-}$.

We now show that $\xi$ is a decreasing function on $\operatorname{Int} X$. Suppose not. Then there exist $0<a<b<1$ such that $\xi(a) \geq \xi(b)$. Because $\xi, \xi^{-}$and $\xi^{+}$are non-increasing functions, all three must be constant on the interval $[a, b]$. From the fact that $\xi^{-}$is constant on $[a, b]$ it follows that $[a, b] \subset X_{-}^{c}$, while from the fact that $\xi^{+}$is constant it follows that $[a, b] \subset X_{+}^{c}$. This contradiction establishes that $\xi$ is a decreasing function on $\operatorname{Int} X$.

Finally, $\xi$ is a decreasing function on $X$ because $\xi(0)=+\infty$ and $\xi(1)=-\infty$.

Definition 6 The point $x \in X$ is a generalized zero of the function $f: X \rightarrow \mathbb{R} \cup$ $\{-\infty,+\infty\}$ if there are sequences $x_{-}^{n}$ and $x_{+}^{n}$ of points in $X$ converging to $x$ such that $\lim f\left(x_{-}^{n}\right) \leq 0 \leq \lim f\left(x_{+}^{n}\right)$.

It is clear that any point $x \in X$ such that $f(x)=0$ is a generalized zero of the function $f$ (take $x_{-}^{n}=x$ and $x_{+}^{n}=x$ ). Conversely, if $f$ is a continuous function, then each generalized zero $x$ of $f$ satisfies $f(x)=0$. If $f$ is a decreasing function, it has at most one generalized zero. We are now in a position to state the main result of the paper.

Theorem 4 (Main result) Each world $\omega$ has a unique bargaining outcome. The bargaining outcome of $\omega$ is the unique generalized zero of the characteristic function $\xi$.

Before we proceed with the proof, we verify for the world $\lambda$ of Section 3 that the conclusion of Proposition 1 agrees with the conclusion of Theorem 4 . Thus let $\lambda$ be the world as in Section 3. Let $g(x)=\mu(\{i \in N \mid x<i\})$. Recall that Proposition 1 claims the unique bargaining outcome of the world $\lambda$ to be a generalized fixed point $x_{*}$ of the function $g$. 
Proposition 7 The characteristic function of the world $\lambda$ can be written as

$$
\xi(x)=\frac{g(x)-x \mu(N \backslash\{x\})}{x(1-x)}
$$

in the interior of $X$. The generalized fixed point $x_{*}$ of the function $g$ is the generalized zero of the function $\xi$.

Proof. We compute:

$$
\frac{u_{t}^{\prime}(x \pm 0)}{u_{t}(x)}= \begin{cases}-1 /(1-x+t) & \text { if } t<x \\ \mp 1 & \text { if } t=x \\ 1 /(1-t+x) & \text { if } x<t\end{cases}
$$

Notice that for a fixed $x \in \operatorname{Int} X$ the functions $u_{t}^{\prime}(x \pm 0) / u_{t}(x)$ are non-decreasing in $t$. Therefore for each $x \in \operatorname{Int} X$ we have

$$
\begin{aligned}
\varphi^{-}(x)=-\frac{1}{1-x}, \quad \varphi^{+}(x)=\frac{1}{x} \\
\xi(x)=-\frac{\mu(\{i \in N \mid i<x\})}{1-x}+\frac{\mu(\{i \in N \mid x<i\})}{x}= \\
=\frac{\mu(\{i \in N \mid x<i\})-x \mu(N \backslash\{x\})}{x(1-x)}= \\
=\frac{g(x)-x \mu(N \backslash\{x\})}{x(1-x)} .
\end{aligned}
$$

We argue that $g\left(x^{\prime}\right) \leq x_{*} \leq g\left(x^{\prime \prime}\right)$ whenever $x^{\prime}$ and $x^{\prime \prime}$ are points of $X$ such that $x^{\prime \prime}<x_{*}<x^{\prime}$. To see this, let the sequences $x_{-}^{n}$ and $x_{+}^{n}$ be as in Definition 4 . Then $x^{\prime \prime}<x_{+}^{n}$ and $x_{-}^{n}<x^{\prime}$ for $n$ large. Since $g$ is a non-increasing function, we have $g\left(x^{\prime}\right) \leq g\left(x_{-}^{n}\right)$ and $g\left(x_{+}^{n}\right) \leq g\left(x^{\prime \prime}\right)$. Therefore, we also have $g\left(x^{\prime}\right) \leq \lim g\left(x_{-}^{n}\right) \leq x_{*} \leq \lim g\left(x_{+}^{n}\right) \leq g\left(x^{\prime \prime}\right)$.

First we consider the case where $x_{*}$ lies on the boundary of $X$. Suppose $x_{*}=0$. It follows from the previous paragraph that $g(x) \leq 0$ whenever $0<x \leq 1$. Therefore $\xi(x) \leq 0$ for all $x \in \operatorname{Int} X$. Since $\xi(0)=+\infty$, it follows that 0 is a generalized fixed point of the function $\xi$, as desired.

If $x_{*}=1$, then $1 \leq g(x)$ whenever $0 \leq x<1$. As $x \mu(N \backslash\{x\}) \leq 1$ it follows that $0 \leq \xi(x)$ for all $0 \leq x<1$. Since $\xi(1)=-\infty$, the point 1 is a generalized fixed point of $\xi$.

Finally, consider the case where $x_{*}$ is in the interior of $X$. Let $z_{+}^{n}=x-1 / n$ and $z_{-}^{n}=x+1 / n$. Notice that $g\left(z_{-}^{n}\right) \leq x_{*} \leq g\left(z_{+}^{n}\right)$ for all $n$ because $z_{+}^{n}<x_{*}<z_{-}^{n}$. Therefore, $\lim g\left(z_{-}^{n}\right) \leq x_{*} \leq \lim g\left(z_{+}^{n}\right)$

We argue that $\lim \mu\left(\left\{z_{-}^{n}\right\}\right)=0$ and $\lim \mu\left(\left\{z_{+}^{n}\right\}\right)=0$. Suppose the first equation is false. Replacing, if necessary, the sequence $\left\{z_{-}^{n}\right\}$ by a subsequence we have $\mu\left(\left\{z_{-}^{n}\right\}\right)>\epsilon$. However, 
since no two points in the sequence $\left\{z_{-}^{n}\right\}$ are the same, $\mu\left(\cup\left\{z_{-}^{n}\right\}\right)=\sum \mu\left(\left\{z_{-}^{n}\right\}\right) \geq+\infty$, a contradiction. It follows that $\lim \mu\left(N \backslash\left\{z_{-}^{n}\right\}\right)=1$ and $\lim \mu\left(N \backslash\left\{z_{+}^{n}\right\}\right)=1$.

Now, we compute

$$
\begin{aligned}
& \lim \xi\left(z_{-}^{n}\right)=\frac{\lim g\left(z_{-}^{n}\right)-x_{*}}{x_{*}\left(1-x_{*}\right)} \leq 0, \\
& \lim \xi\left(z_{+}^{n}\right)=\frac{\lim g\left(z_{+}^{n}\right)-x_{*}}{x_{*}\left(1-x_{*}\right)} \geq 0,
\end{aligned}
$$

establishing that $x_{*}$ is a generalized zero of $\xi$.

\section{$7 \quad$ A proof of the main result}

Proposition 8 Let $f: X \rightarrow \mathbb{R}$ be a non-negative concave function. Suppose that $f$ is positive on the interior of $X$.

(a) For each $x \in \operatorname{Int} X$ the following inequalities hold:

$$
-\frac{1}{1-x} \leq \frac{f^{\prime}(x+0)}{f(x)} \leq \frac{f^{\prime}(x-0)}{f(x)} \leq \frac{1}{x}
$$

(b) Given an $x \in X$ and a number $0<\kappa<1$ define the sets $I(x, \kappa)$ and $B(x, \kappa)$ by the following equations:

$$
\begin{gathered}
I(x, \kappa)=\left[\frac{x}{1-\ln \kappa}, \frac{x-\ln \kappa}{1-\ln \kappa}\right], \\
B(x, \kappa)=\{\dot{x} \in X \mid f(\dot{x}) \geq \kappa f(x)\} .
\end{gathered}
$$

Then $I(x, \kappa) \subset B(x, \kappa)$.

Proof. Consider a chain of inequalities

$$
-\frac{f(x)}{1-x} \leq \frac{f(1)-f(x)}{1-x} \leq f^{\prime}(x+0) \leq f^{\prime}(x-0) \leq \frac{f(x)-f(0)}{x} \leq \frac{f(x)}{x} .
$$

Dividing by $f(x)$ yields the inequalities of claim (a). Consider a concave function $\ell=\ln f$ : Int $X \rightarrow \mathbb{R}$. We have

$$
\ell^{\prime}(x+0)=\frac{f^{\prime}(x+0)}{f(x)} \text { and } \ell^{\prime}(x-0)=\frac{f^{\prime}(x-0)}{f(x)} .
$$

Given a point $\bar{x} \in X$, define a function $h: \operatorname{Int} X \rightarrow \mathbb{R}$ by the equation

$$
h(x)= \begin{cases}\frac{x-\bar{x}}{x} & \text { if } x \leq \bar{x} \\ \frac{\bar{x}-x}{1-x} & \text { if } \bar{x} \leq x .\end{cases}
$$


The function $h$ is non-positive and concave and it attains the value of zero at point $\bar{x}$. Then for each $x \in \operatorname{Int} X$ we have the inequalities $h(x) \leq \ell^{\prime}(x+0)(x-\bar{x}) \leq \ell(x)-\ell(\bar{x})$, where the first inequality can be established using claim (a).

Both intervals $I(\bar{x}, \kappa)$ and $B(\bar{x}, \kappa)$ contain the point $\bar{x}$, and the interval $I(\bar{x}, \kappa)$ is nondegenerate. To prove the inclusion $I(\bar{x}, \kappa) \subset B(\bar{x}, \kappa)$, it is sufficient to prove that either endpoint of $I(\bar{x}, \kappa)$ is contained in $B(\bar{x}, \kappa)$. Thus let $\dot{x}$ be an endpoint of $I(\bar{x}, \kappa)$. If $\dot{x}=0$, then $\bar{x}=0$, and if $\dot{x}=1$, then $\bar{x}=1$. In either case the point $\dot{x}=\bar{x}$ is an element of $B(\bar{x}, \kappa)$. If $\dot{x}$ is in the interior of $X$, it is straightforward to verify that $h(\dot{x})=\ln \kappa$. Therefore $\ln \kappa \leq \ell(\dot{x})-\ell(\bar{x})$, implying that $\kappa f(\bar{x}) \leq f(\dot{x})$, as desired.

\section{Proposition 9}

(a) For each $x \in \operatorname{Int} X$ the inequalities $-1 /(1-x) \leq \varphi^{-}(x) \leq \varphi^{+}(x) \leq 1 / x$ hold.

(b) Suppose $\delta \in(0,1)$ and let $\left(x_{\bullet}, y_{\bullet}, A_{\bullet}, A\right)$ be a $\delta$-equilibrium. Then $I\left(E\left(x_{\bullet}\right), \delta\right) \subset A$. Consequently, $A$ is a non-degenerate interval.

Proof. Claim (a) of Proposition 9 follows immediately from claim (a) of Proposition 8. Consider the sets

$$
B_{t}=\left\{x \in X \mid u_{t}(x) \geq \delta u_{t}\left(E\left(x_{\bullet}\right)\right)\right\} .
$$

By claim (b) of Proposition $8, I\left(E\left(x_{\bullet}\right), \delta\right) \subset B_{t}$ for each $t$. One the other hand $B_{t} \subset A_{t}$, because by Jensen inequality $u_{t}\left(E\left(x_{\bullet}\right)\right) \geq E\left(u_{t}\left(x_{\bullet}\right)\right)=y_{t}$. The result follows.

Proposition 10 Suppose $\delta \in(0,1)$ and let $\left(x_{\bullet}, y_{\bullet}, A_{\bullet}, A\right)$ be a $\delta$-equilibrium. Let $A_{t}=$ $\left[x_{t}^{-}, x_{t}^{+}\right]$and $A=\left[x^{-}, x^{+}\right]$. If $x^{-} \in \operatorname{Int} X$, then

$$
\sup _{x^{-}<\bar{x}_{t}}\left\{\delta y_{t} / u_{t}\left(x^{-}\right)\right\}=1,
$$

where the supremum is taken over all players $t \in N$ with $x^{-}<\bar{x}_{t}$. If $x^{+} \in$ Int $X$, then

$$
\sup _{\bar{x}_{t}<x^{+}}\left\{\delta y_{t} / u_{t}\left(x^{+}\right)\right\}=1 \text {, }
$$

where the supremum is taken over all players $t \in N$ with $\bar{x}_{t}<x^{+}$.

Proof. We only prove the first claim, the proof of the second claim being similar. 
Let $r_{t}^{-}=\delta y_{t} / u_{t}\left(x^{-}\right)$. We know that $r_{t}^{-} \leq 1$ for all $t$. Take a player $t$ such that $x_{t}^{-}$is in the interior of $X$. Then $u_{t}\left(x_{t}^{-}\right)=\delta y_{t}$ and we have the following chain of inequalities:

$$
\begin{aligned}
0 \leq 1-r_{t}^{-} & =1-\frac{u_{t}\left(x_{t}^{-}\right)}{u_{t}\left(x^{-}\right)}= \\
& =\frac{u_{t}\left(x^{-}\right)-u_{t}\left(x_{t}^{-}\right)}{u_{t}\left(x^{-}\right)} \leq \\
& \leq \frac{u_{t}\left(x^{-}\right)-u_{t}\left(x_{t}^{-}\right)}{u_{t}\left(x_{t}^{-}\right)} \leq \\
& \leq \frac{u_{t}^{\prime}\left(x_{t}^{-}+0\right)}{u_{t}\left(x_{t}^{-}\right)}\left(x^{-}-x_{t}^{-}\right) \leq \\
& \leq \frac{x^{-}-x_{t}^{-}}{x_{t}^{-}},
\end{aligned}
$$

where the last inequality follows from claim (a) of Proposition 8.

First we show that $\sup \left\{r_{t}^{-}\right\}=1$, where the supremum is taken over all players is $N$. Since $x^{-}=\sup \left\{x_{t}^{-}\right\}$there is an increasing sequence $x_{t^{n}}^{-}$converging to $x^{-}$. Of course, $x_{t^{n}}^{-}$is in the interior of $X$ for $n$ large enough, so the inequalities of the previous paragraph apply. In particular,

$$
0 \leq 1-r_{t^{n}}^{-} \leq \frac{x^{-}-x_{t^{n}}^{-}}{x_{t^{n}}^{-}}
$$

Since the sequence on the right side converges to zero, the sequence $r_{t^{n}}^{-}$converges to one. This proves that $\sup \left\{r_{t}^{-}\right\}=1$.

Now, for any player $t$ with $\bar{x}_{t} \leq x^{-}$the function $u_{t}$ is non-increasing on the interval $A=\left[x^{-}, x^{+}\right]$. In particular, $u_{t}(x) \leq u_{t}\left(x^{-}\right)$for any $x \in A$. It follows that $y_{t} \leq u_{t}\left(x^{-}\right)$, since $y_{t}$ is an integral of $u_{t}\left(x_{i}\right)$ where each $x_{i}$ is a point of $A$. Thus $r_{t}^{-} \leq \delta$ for any $t$ with $\bar{x}_{t} \leq x^{-}$. This proves that $\sup _{x^{-}<\bar{x}_{t}}\left\{r_{t}^{-}\right\}=1$.

Proposition 11 Suppose $\delta \in(0,1)$. Let $\left(x_{\bullet}, y_{\bullet}, A_{\bullet}, A\right)$ be a $\delta$-equilibrium, where $A=$ $\left[x^{-}, x^{+}\right]$. If $x^{+} \in \operatorname{Int} X$, then the following inequalities hold:

$$
\begin{gathered}
\delta \mu\left(x^{+}<\bar{x}_{i}\right) \varphi^{+}\left(x^{+}\right) \leq \frac{1-\delta}{x^{+}-x^{-}} \\
\delta \mu\left(\bar{x}_{i}<x^{+}\right) \varphi^{-}\left(x^{+}\right) \leq-\frac{1-\delta}{x^{+}-x^{-}} .
\end{gathered}
$$

If $x^{-} \in \operatorname{Int} X$, then, the following inequalities hold:

$$
\begin{gathered}
-\frac{1-\delta}{x^{+}-x^{-}} \leq \delta \mu\left(\bar{x}_{i}<x^{-}\right) \varphi^{-}\left(x^{-}\right) \\
\frac{1-\delta}{x^{+}-x^{-}} \leq \delta \mu\left(x^{-}<\bar{x}_{i}\right) \varphi^{+}\left(x^{-}\right)
\end{gathered}
$$


Proof. Suppose that $x^{+} \in \operatorname{Int} X$. We prove the first inequality of Proposition 11. Let $x^{+} \leq \bar{x}_{t}$. First we snow that

$$
\mu\left(x^{+}<\bar{x}_{i}\right)\left[u_{t}\left(x^{+}\right)-u_{t}\left(x^{-}\right)\right] \leq y_{t}-u_{t}\left(x^{-}\right) .
$$

Too see this, for $i \in N$ define $\hat{x}_{i}$ to be $x^{-}$if $\bar{x}_{i} \leq x^{+}$and $x^{+}$otherwise. Clearly, $\hat{x}_{i}$ is a measurable function of $i$. Furthermore, $\hat{x}_{i} \leq x_{i}$ for all $i \in N$. As the function $u_{t}$ is nondecreasing on the interval $\left[x^{-}, x^{+}\right]$, we have the inequality $u_{t}\left(\hat{x}_{i}\right) \leq u_{t}\left(x_{i}\right)$ for all $i \in N$. The integration gives $\mu\left(\bar{x}_{i} \leq x^{+}\right) u_{t}\left(x^{-}\right)+\mu\left(x^{+}<\bar{x}_{i}\right) u_{t}\left(x^{+}\right) \leq y_{t}$. Subtracting $u_{t}\left(x^{-}\right)$from both sides yields the desired inequality.

As before, let $x^{+} \leq \bar{x}_{t}$. From the fact that $\delta y_{t} \leq u_{t}\left(x^{-}\right) \leq u_{t}\left(x^{+}\right)$it follows that

$$
\delta\left[y_{t}-u_{t}\left(x^{-}\right)\right] \leq(1-\delta) u_{t}\left(x^{+}\right)
$$

Finally, we use the inequality

$$
u_{t}^{\prime}\left(x^{+}+0\right) \leq \frac{u_{t}\left(x^{+}\right)-u_{t}\left(x^{-}\right)}{x^{+}-x^{-}} .
$$

Combining them all, we conclude that that for any player $t$ with $x^{+} \leq \bar{x}_{t}$

$$
\begin{aligned}
\delta \mu\left(x^{+}<\bar{x}_{i}\right) \frac{u_{t}^{\prime}\left(x^{+}+0\right)}{u_{t}\left(x^{+}\right)} & \leq \delta \mu\left(x^{+}<\bar{x}_{i}\right) \frac{\left[u_{t}\left(x^{+}\right)-u_{t}\left(x^{-}\right)\right]}{u_{t}\left(x^{+}\right)\left[x^{+}-x^{-}\right]} \leq \\
& \leq \delta \frac{y_{t}-u_{t}\left(x^{-}\right)}{u_{t}\left(x^{+}\right)\left[x^{+}-x^{-}\right]} \leq \\
& \leq \frac{1-\delta}{x^{+}-x^{-}} .
\end{aligned}
$$

For a player $t$ with $\bar{x}_{t} \leq x^{+}$the expression on the extreme left-hand side of the last inequality is non-positive, because $u_{t}^{\prime}\left(x^{+}+0\right) \leq 0$. This proves the first inequality of Proposition 11.

We now prove the second inequality of Proposition 11. Let $\bar{x}_{t}<x^{+}$. First we show that

$$
\mu\left(\bar{x}_{i}<x^{+}\right)\left[u_{t}\left(x^{+}\right)-u_{t}\left(x_{t}\right)\right] \leq u_{t}\left(x^{+}\right)-y_{t} .
$$

To see this, for $i \in N$ define $\hat{x}_{i}$ to be $x_{t}$ if $\bar{x}_{i}<x^{+}$and $x^{+}$otherwise. Since $x_{t}$ is the maximum of $u_{t}$ on $[0,1], u_{t}\left(x_{i}\right) \leq u_{t}\left(\hat{x}_{i}\right)$ for all $i \in N$. The integration yields $y_{t} \leq \mu\left(\bar{x}_{i}<\right.$ $\left.x^{+}\right) u_{t}\left(x_{t}\right)+\mu\left(x^{+} \leq \bar{x}_{i}\right) u_{t}\left(x^{+}\right)$. Subtracting $u_{t}\left(x^{+}\right)$from both sides and multiplying by minus one give the desired inequality.

Now since $x^{-} \leq x_{t}<x^{+}$, we have

$$
u_{t}^{\prime}\left(x^{+}-0\right) \leq \frac{u_{t}\left(x^{+}\right)-u_{t}\left(x_{t}\right)}{x^{+}-x_{t}} \leq \frac{u_{t}\left(x^{+}\right)-u_{t}\left(x_{t}\right)}{x^{+}-x^{-}} \leq 0 .
$$


Thus for each $t$ with $\bar{x}_{t}<x^{+}$we have the inequalities

$$
\begin{aligned}
\delta \mu\left(\bar{x}_{i}<x^{+}\right) \frac{u_{t}^{\prime}\left(x^{+}-0\right)}{u_{t}\left(x^{+}\right)} & \leq \delta \mu\left(\bar{x}_{i}<x^{+}\right) \frac{\left[u_{t}\left(x^{+}\right)-u_{t}\left(x_{t}\right)\right]}{u_{t}\left(x^{+}\right)\left[x^{+}-x^{-}\right]} \leq \\
& \leq \delta \frac{u_{t}\left(x^{+}\right)-y_{t}}{u_{t}\left(x^{+}\right)\left[x^{+}-x^{-}\right]}= \\
& =-\frac{r_{t}^{+}-\delta}{x^{+}-x^{-}}
\end{aligned}
$$

where $r_{t}^{+}=\delta y_{t} / u_{t}\left(x^{+}\right)$. Using Proposition 10, we obtain

$$
\begin{aligned}
\delta \mu\left(\bar{x}_{i}<x^{+}\right) \varphi^{-}\left(x^{+}\right) & \leq \delta \mu\left(\bar{x}_{i}<x^{+}\right) \inf _{\bar{x}_{t}<x^{+}}\left\{\frac{u_{t}^{\prime}\left(x^{+}-0\right)}{u_{t}\left(x^{+}\right)}\right\} \leq \\
& \leq \inf _{\bar{x}_{t}<x^{+}}\left\{-\frac{r_{t}^{+}-\delta}{x^{+}-x^{-}}\right\}= \\
& =-\frac{1-\delta}{x^{+}-x^{-}} .
\end{aligned}
$$

Suppose that $x^{-} \in \operatorname{Int} X$. We now prove the third inequality of Proposition 11. This proof is similar to the proof of the first inequality. Let $\bar{x}_{t} \leq x^{-}$. First we show that

$$
u_{t}\left(x^{+}\right)-y_{t} \leq \mu\left(\bar{x}_{i}<x^{-}\right)\left[u_{t}\left(x^{+}\right)-u_{t}\left(x^{-}\right)\right] .
$$

To prove this, notice that the function $u_{t}$ is non-increasing on the interval $\left[x^{-}, x^{+}\right]$. The expected utility of player $t$ can therefore be estimated from below as $\mu\left(\bar{x}_{i}<x^{-}\right) u_{t}\left(x^{-}\right)+$ $\mu\left(x^{-} \leq \bar{x}_{i}\right) u_{t}\left(x^{+}\right) \leq y_{t}$. Subtracting $u_{t}\left(x^{+}\right)$from both sides and multiplying by minus one yields the desired inequality. Secondly, the fact that $\delta y_{t} \leq u_{t}\left(x^{+}\right) \leq u_{t}\left(x^{-}\right)$implies the inequality

$$
-(1-\delta) u_{t}\left(x^{-}\right) \leq \delta\left[u_{t}\left(x^{+}\right)-y_{t}\right]
$$

Thirdly, we have

$$
\frac{u_{t}\left(x^{+}\right)-u_{t}\left(x^{-}\right)}{x^{+}-x^{-}} \leq u_{t}^{\prime}\left(x^{-}-0\right)
$$

Combining these three inequalities, we conclude that for each player $t$ with $\bar{x}_{t} \leq x^{-}$

$$
-\frac{1-\delta}{x^{+}-x^{-}} \leq \delta \mu\left(\bar{x}_{i}<x^{-}\right) \frac{u_{t}^{\prime}\left(x^{-}-0\right)}{u_{t}\left(x^{-}\right)} .
$$

This inequality also holds if $x^{-}<\bar{x}_{t}$ since in this case $0 \leq u_{t}^{\prime}\left(x^{-}-0\right)$. This proves the third inequality of Proposition 11.

The proof of the fourth inequality is similar to the proof of the second one. Let $x^{-}<\bar{x}_{t}$. First, we establish

$$
y_{t}-u_{t}\left(x^{-}\right) \leq \mu\left(x^{-}<\bar{x}_{i}\right)\left[u_{t}\left(x_{t}\right)-u_{t}\left(x^{-}\right)\right]
$$


This is done by estimating thew expected utility of player $t$ from above, as follows: $y_{t} \leq$ $\mu\left(\bar{x}_{i} \leq x^{-}\right) u_{t}\left(x^{-}\right)+\mu\left(x^{-}<\bar{x}_{i}\right) u_{t}\left(x_{t}\right)$, and then subtracting $u_{t}\left(x^{-}\right)$from both sides. Since $x^{-}<x_{t} \leq x^{+}$, we have

$$
0 \leq \frac{u_{t}\left(x_{t}\right)-u_{t}\left(x^{-}\right)}{x^{+}-x^{-}} \leq \frac{u_{t}\left(x_{t}\right)-u_{t}\left(x^{-}\right)}{x_{t}-x^{-}} \leq u_{t}^{\prime}\left(x^{-}+0\right) .
$$

Thus for each player $t$ with $x^{-}<\bar{x}_{t}$ we have the inequalities

$$
\begin{aligned}
\frac{r_{t}^{-}-\delta}{x^{+}-x^{-}} & =\delta \frac{y_{t}-u_{t}\left(x^{-}\right)}{u_{t}\left(x^{-}\right)\left[x^{+}-x^{-}\right]} \leq \\
& \leq \delta \mu\left(x^{-}<\bar{x}_{i}\right) \frac{\left[u_{t}\left(x_{t}\right)-u_{t}\left(x^{-}\right)\right]}{u_{t}\left(x^{-}\right)\left[x^{+}-x^{-}\right]} \leq \\
& \leq \delta \mu\left(x^{-}<\bar{x}_{i}\right) \frac{u_{t}^{\prime}\left(x^{-}+0\right)}{u_{t}\left(x^{-}\right)} .
\end{aligned}
$$

Finally, using Proposition 10, we obtain

$$
\begin{aligned}
\frac{1-\delta}{x^{+}-x^{-}} & =\sup _{x^{-}<\bar{x}_{t}}\left\{\frac{r_{t}^{-}-\delta}{x^{+}-x^{-}}\right\} \leq \\
& \leq \delta \mu\left(x^{-}<\bar{x}_{i}\right) \sup _{x^{-}<\bar{x}_{t}}\left\{\frac{u_{t}^{\prime}\left(x^{-}+0\right)}{u_{t}\left(x^{-}\right)}\right\} \\
& \leq \delta \mu\left(x^{-}<\bar{x}_{i}\right) \varphi^{+}\left(x^{-}\right) .
\end{aligned}
$$

This completes the proof of Proposition 11.

Proposition 12 Each bargaining outcome of the world $\omega$ is a generalized zero point of the characteristic function $\xi$.

Proof. Let $x$ be a bargaining outcome of $\omega$. By Definition 3, there exist sequences $A^{n}$, $\delta^{n}$, and $x^{n}$ such that $A^{n}$ is a social acceptance set in a $\delta^{n}$-equilibrium of $\omega, x^{n}$ is a point in $A^{n}$, the sequence $\delta^{n}$ converges to 1 and $x^{n}$ converges to $x$. Let $A^{n}=\left[x_{-}^{n}, x_{+}^{n}\right]$. Replacing, if necessary, sequences by subsequences, assume that both $x_{-}^{n}$ and $x_{+}^{n}$ converge. Then Theorem 3 implies that both sequences converge to $x$.

First suppose that $x \in \operatorname{Int} X$. Then $x_{-}^{n}$ and $x_{+}^{n}$ both lie in $\operatorname{Int} X$ for $n$ large enough. The first two inequalities in Proposition 11 then imply that $\xi\left(x_{+}^{n}\right) \leq 0$ while the last two imply $0 \leq \xi\left(x_{-}^{n}\right)$. It follows that $x$ is generalized fixed point of the function $\xi$.

Now suppose $x=0$. Then $x_{+}^{n}<1$ for $n$ large enough. Since $A^{n}$ is a non-degenerate interval by Proposition 9, we have $0<x_{+}^{n}$. Then again the first two inequalities in Proposition 11 imply that $\xi\left(x_{+}^{n}\right) \leq 0$. Since $\xi(0)=+\infty$, it follows that $x$ is a generalized fixed point of $\xi$. The argument in the case $x=1$ is similar. 


\section{The analysis of the game}

We start our analysis by computing the payoffs induced by a joint stationary strategy $\sigma=\left(x_{\bullet}, A_{\bullet}\right)$. Let $y_{t}$ denote the expected payoff to player $t$ at the beginning of the game, let $A=\cap A_{t}$ be a social acceptance set and $N_{a}$ be the set of players whose proposal is accepted under $\sigma$ i.e. $N_{a}=\left\{t \in N \mid x_{t} \in A\right\}$. Let $N_{r}$ denote the set of players whose proposal is rejected, i.e. the complement of $N_{a}$. Notice that $A$ is measurable set as it is an intersection of measurable sets, and the set $N_{a}$ is $\mathscr{A}$-measurable as it is a preimage of an $\mathscr{A}$-measurable set under an $\mathscr{A}$-measurable map. If nature chooses a proposer $i$ from $N_{a}$, player $t$ receives a payoff of $u_{t}\left(x_{i}\right)$, while if nature chooses a proposer from $N_{r}$, then player $t$ 's payoff is $\delta y_{t}$. Thus $y_{t}$ satisfies the following relation:

$$
y_{t}=\int_{N_{a}} u_{t}\left(x_{i}\right) d \mu(i)+\delta \mu\left(N_{r}\right) y_{t}
$$

Solving the equation, we obtain the following expression for $y_{t}$ :

$$
y_{t}= \begin{cases}\frac{1}{1-\delta \mu\left(N_{r}\right)} \int_{N_{a}} u_{t}\left(x_{i}\right) d \mu(i) & \text { if } \mu\left(N_{a}\right)>0 \\ 0 & \text { otherwise }\end{cases}
$$

Of course, we are only interested in admissible, that is joint strategies that result in a well-defined payoff to each player at any node of the game. While any joint stationary strategy induces a well-defined payoff at each node of the game, finding sufficient conditions for a general joint strategy to be admissible is a difficult problem on its own and it is certainly beyond the scope of this paper. In what follows, we simply assume that all joint strategies involved are admissible.

Theorem 5 Let $\left(x_{\bullet}, y_{\bullet}, A_{\bullet}, A\right)$ be a $\delta$-equilibrium. Then a joint stationary strategy $\sigma=$ $\left(x_{\bullet}, A_{\bullet}\right)$ is a subgame perfect equilibrium of $\Gamma(\delta)$.

The proof of Theorem 5 consists of two steps. The first step is to show that strategy $\sigma$ is robust to all one-shot deviations. Recall that a strategy $\bar{\sigma}_{t}$ for player $t$ is said to be a one-shot deviation from $\sigma$ at node $h$ if it coincides with the strategy $\sigma_{t}$ on all nodes but $h$. The second step establishes the one-shot deviation property for the game $\Gamma(\delta)$. The property states that if there is a profitable deviation from a joint strategy $\sigma$, then there is a profitable one-shot deviation. The one-shot deviation property is well-known for finite games, i.e. for games with a finite game tree and finitely many players and less so for infinite games such as $\Gamma(\delta)$.

Proposition 13 Let $\left(x_{\bullet}, y_{\bullet}, A_{\bullet}, A\right)$ be a $\delta$-equilibrium. Let $\sigma$ denote a joint stationary strategy $\left(x_{\bullet}, A_{\bullet}\right)$. Then no player has a one-shot profitable deviation from $\sigma_{t}$ in any subgame of $\Gamma=\Gamma(\delta)$. 
Proof. Let $\bar{\sigma}_{t}$ be a one-shot deviation from $\sigma_{t}$ in a subgame $\Gamma(h)$ of $\Gamma$ starting at node $h$.

Suppose player $t$ has to make a proposal at node $h$. Under strategy $\sigma_{t}$ player $t$ proposes alternative $x_{t}$, which is accepted, leading to a payoff of $u_{t}\left(x_{t}\right)$ for player $t$. Suppose under strategy $\bar{\sigma}_{t}$ player $t$ makes a proposal $x$. If $x$ is not an element of the social acceptance set $A=\cap A_{i}$, the proposal $x$ will be rejected. As $\bar{\sigma}_{t}$ coincides with $\sigma_{t}$ on all nodes following $h$, player $t$ will receive a payoff of $\delta y_{t}$. By the definition of $\delta$-equilibrium, $\delta y_{t} \leq u_{t}\left(x_{t}\right)$. If $x$ is an element of $A$, then $x$ it is accepted and player $t$ receives a payoff of $u_{t}(x)$. However, $u_{t}(x) \leq u_{t}\left(x_{t}\right)$, because by the definition of $\delta$-equilibrium, $x_{t}$ maximizes the function $u_{t}$ on the set $A$.

Suppose player $t$ has to react to a proposal $x$ at node $h$. Suppose $x$ is accepted by player $t$ under strategy $\sigma_{t}$ but rejected under strategy $\bar{\sigma}_{t}$. Then strategy $\sigma_{t}$ leads to a payoff of either $u_{t}(x)$ or $\delta y_{t}$, depending on whether other players accept or reject $x$, while strategy $\bar{\sigma}_{t}$ leads to payoff of $\delta y_{t}$. Then $\bar{\sigma}_{t}$ is not a profitable deviation, because $\delta y_{t} \leq u_{t}(x)$, since $x \in A_{t}$. Conversely, suppose $x$ is rejected by player $t$ under strategy $\sigma_{t}$ but is accepted under strategy $\bar{\sigma}_{t}$. Then strategy $\sigma_{t}$ leads to a payoff of $\delta y_{t}$, while strategy $\bar{\sigma}_{t}$ leads to payoff of either $u_{t}(x)$ or $\delta y_{t}$, depending on whether $x$ is accepted or rejected by other players. Again, $\bar{\sigma}_{t}$ cannot be a profitable deviation, since $u_{t}(x) \leq \delta y_{t}$, as $x$ is not an element of $A_{t}$.

Proposition 14 Let $\sigma$ be a profile of strategies. If player $t$ has a profitable deviation from $\sigma$, then player $t$ has a profitable one-shot deviation from $\sigma$.

Proof. Given a node $h$ we let $\tau(h)$ denote the period node $h$ belongs to. Suppose player $t$ has a profitable deviation $\bar{\sigma}_{t}$ from $\sigma$ in the subgame $\Gamma(\bar{h})$ of the game $\Gamma$ which increases the subgame payoff by $\varepsilon>0$. First we show that player $t$ has a profitable deviation in the subgame $\Gamma(\bar{h})$ that coincides with $\sigma_{t}$ on all nodes corresponding to the first $r$ periods of the subgame $\Gamma(\bar{h})$.

Since the payoff player $t$ can get in period $\tau$ is bounded by $\delta^{\tau}$, the subgame payoff for any strategy that agrees with $\bar{\sigma}_{t}$ on all nodes corresponding to the first $\tau$ periods of the subgame differs from the payoff on $\bar{\sigma}_{t}$ by at most $\delta^{\tau}$. Set $r=\ln (\varepsilon) / \ln (\delta)$ and define the strategy $\sigma_{t}^{r}$ as follows:

$$
\sigma_{t}^{r}(h)= \begin{cases}\bar{\sigma}_{t}(h) & \text { if } \tau(h) \leq r+\tau(\bar{h}) \\ \sigma_{t}(h) & \text { otherwise. }\end{cases}
$$

By definition, $\sigma_{t}^{r}$ coincides with $\bar{\sigma}_{t}$ on all nodes corresponding to the first $r$ periods of the subgame $\Gamma(\bar{h})$. Therefore, the subgame payoff on $\sigma_{t}^{r}$ differs from the payoff on $\bar{\sigma}_{t}$ by $\delta^{r}=\varepsilon$ at the most. It follows that $\sigma_{t}^{r}$ is a profitable deviation from $\sigma_{t}$ in the subgame $\Gamma(\bar{h})$.

Suppose there is a node $h$ in the subgame $\Gamma(\bar{h})$ with $\tau(h)=r+\tau(\bar{h})$ such that player $t$ has to act at $h$ and strategy $\sigma_{t}^{r}$ is a profitable deviation from $\sigma$ in the subsubgame $\Gamma(h)$. Then $\sigma_{t}^{r}$ is a profitable one-shot deviation in the subsubgame $\Gamma(h)$. This follows from the fact that each player acts at most once every period. Thus if node $h^{\prime}$ follows node $h$ and player $t$ has to act at $h^{\prime}$, then $\tau\left(h^{\prime}\right)>r+\tau(\bar{h})$, implying that $\sigma_{t}^{r}\left(h^{\prime}\right)=\sigma_{t}\left(h^{\prime}\right)$. In this case the argument is complete. 
Suppose there is no node $h$ in the subgame $\Gamma(\bar{h})$ with $\tau(h)=r+\tau(\bar{h})$ such that player $t$ has to act at $h$ and strategy $\sigma_{t}^{r}$ is a profitable deviation in the subsubgame $\Gamma(h)$. In this case we define a new strategy for $t$ as follows:

$$
\sigma_{t}^{r-1}(h)= \begin{cases}\sigma_{t}^{r}(h) & \text { if } \tau(h) \leq r+\tau(\bar{h})-1 \\ \sigma_{t}(h) & \text { otherwise. }\end{cases}
$$

Then the payoff on $\sigma_{t}^{r-1}$ at any node of the subgame $\Gamma(\bar{h})$ is at least as high as the payoff on $\sigma_{t}^{r}$. In particular, $\sigma_{t}^{r-1}$ is a profitable deviation from $\sigma$ in the subgame $\Gamma(\bar{h})$. Iterating this argument, we shall be able to find a one-shot profitable deviation for player $t$.

We now turn to the converse of Theorem 5. Before we state the result a few remarks are in order concerning players' equilibrium behavior in the response stage of the game.

It is clear that individual acceptance sets are not uniquely determined. Take a subgame perfect equilibrium $\sigma=\left(x_{\bullet}, A_{\bullet}\right)$. If alternative $x$ is not accepted by player $t\left(x \notin A_{t}\right)$, each player $i$ preceding player $t$ in the response stage is indifferent between accepting and rejecting $x$, since by the stationarity of $\sigma$ the continuation payoff is the same whether $x$ is rejected by player $i$ or player by $t$. Starting from $\sigma$, one can obtain a new subgame perfect equilibrium by adding $x$ to or subtracting $x$ from the individual acceptance set $A_{i}$ of player $i<t$. The profile of strategies thus obtained is a subgame perfect equilibrium and it is equivalent to $\sigma$ in the sense that the proposals, the social acceptance set and equilibrium payoffs are the same.

On top of the indeterminateness of the individual acceptance sets, when there are infinitely many players, coordination failure might occur in the response stage. The coordination failure may be present in equilibrium despite the fact that the players react to a proposal sequentially.

As an example, consider a world with a set of players $N=[0,1]$. Suppose that the order in which the players react during the response stage is the natural order on $N$, i.e. player 0 reacts first, player 1 reacts last. Let $\iota$ be a sequence in $N$ converging to 1 . Let $C$ be a subset of players $\cup \iota_{n}$. Then any joint strategy $\sigma=\left(x_{\bullet}, A_{\bullet}\right)$ is a subgame perfect equilibrium, provided only that the sets $A_{t}$ are empty for each $t \in C$ and $A_{1}=X$. The condition that $A_{t}$ is empty means that player $t$ rejects all proposals, while $A_{1}=X$ means that player 1 accepts all proposals. Of course, $\sigma$ induces perpetual disagreement. Each player $t<1$ in the response stage is indifferent between accepting and rejecting a proposal, as player $t$ knows that even if he will accept, a proposal will be later rejected by one of the players in $C$.

It is clear that the situation described above is peculiar to the case where there are infinitely many players. If there were finitely many players, since the last player accepts all proposals, the player who is second-last to respond should accept all proposals with a positive payoff. In the above example there is no second-last player in the sense that each player $t<1$ is succeeded by a member of $C$.

To rule out coordination problems as described above we shall require that if alternative $x$ is rejected, then for at least one player $t$ rejection must be a best response not only to 
the current strategy of his opponents, but also to a modified profile of strategies where all players following $t$ accept a proposal in question, but continue playing according to the original profile later on. The definition is as follows.

Definition 7 Let $\sigma$ be a joint strategy and let $h$ be a history of player ending with player $k$ proposing alternative $x$. Let $h_{t}$ denote the history following $h$ after which player $t$ has to react to the proposal. Let $\sigma_{t}^{a}$ denote a strategy for player $t$ that coincides with $\sigma_{t}$ on all histories other than $h_{t}$ and $\sigma_{t}^{a}\left(h_{t}\right)=a$.

A joint strategy $\sigma$ is said to be free of coordination failures in the response stage if either $x$ is accepted under $\sigma$ or there is a player $t$ with $\sigma_{t}\left(h_{t}\right)=r$ such that the strategy $\sigma_{t}$ is a best response against a profile of strategies $\sigma_{-t}^{a}$ of player $t$ 's opponents in the subgame $\Gamma(\delta)\left(h_{t}\right)$.

A subgame perfect equilibrium $\sigma$ is free of coordination failures in the response stage provided that any rejected alternative is rejected by at most finitely many players. In this case player $t$ who is the last player in the order $<$ to reject $x$ satisfies the requirement of the above definition. In particular, if the player set $N$ is finite, then any subgame perfect equilibrium is free of coordination failures. On the other hand, a joint strategy considered above violates the condition in Definition 7, as each player $t$ should accept any alternative $x$ having positive utility, provided that the following players following $t$ accept.

Theorem 6 Suppose $0 \leq \delta<1$. Consider a joint strategy $\sigma=\left(x_{\bullet}, A_{\bullet}\right)$ with the expected payoff map $y_{\bullet}$ and the social acceptance set $A=\cap A_{t}$. Suppose $\sigma$ is a subgame perfect equilibrium and that it is free of coordination failures in the response stage. Then there exists a $\delta$-equilibrium $\left(x_{\bullet}, y_{\bullet}, B \bullet, B\right)$ such that $\operatorname{Int} B \subset A \subset B$.

Proof. The proof uses Proposition 8 of Section 7.

Define the collections of sets $B_{\bullet}, C_{\bullet}$, and the sets $B$ and $C$ as follows:

$$
\begin{aligned}
& B_{t}=\left\{x \in X \mid u_{t}(x) \geq \delta y_{t}\right\} \text { and } B=\cap B_{t}, \\
& C_{t}=\left\{x \in X \mid u_{t}(x)>\delta y_{t}\right\} \text { and } C=\cap C_{t} .
\end{aligned}
$$

All sets are convex, the set $B$ is closed and $\operatorname{Int} B \subset C$.

First we prove the inclusions $C \subset A \subset B$. To prove the first inclusion, suppose $x$ is not an element of $A$. Let the strategies $\sigma_{\bullet}^{a}$ be as in Definition 7 above. There exists a player $t$ with $\sigma_{t}\left(h_{t}\right)=r$ such that the strategy $\sigma_{t}$ is a best response against a joint strategy $\sigma_{-t}^{a}$ of player $t$ 's opponents at node $h_{t}$. Playing $\sigma_{t}$ when other players use $\sigma_{-t}^{a}$ results leads to a payoff of $\delta y_{t}$ for player $t$. On the other hand, playing $\sigma_{t}^{a}$ against $\sigma_{-t}^{a}$ leads to an acceptance of $x$ and a payoff of $u_{t}(x)$. Since the strategy $\sigma_{t}$ should be at least as good as the strategy $\sigma_{t}^{a}$ at node $h_{t}$, we conclude that $u_{t}(x) \leq \delta y_{t}$. This means that $x \notin C_{t}$ and therefore $x$ is not an element of $C$.

To prove the second inclusion, let $x \in A$. Let $\sigma_{t}^{r}$ denote a strategy for player $t$ that coincides with $\sigma_{t}$ on all histories other than $h_{t}$ and $\sigma_{t}^{r}\left(h_{t}\right)=r$. When other players use 
strategies $\sigma_{-t}$, the payoff on strategy $\sigma_{t}^{r}$ at node $h_{t}$ is $\delta y_{t}$, while the payoff on $\sigma_{t}$ is $u_{t}(x)$. Since $\sigma_{t}$ is at least as good as $\sigma_{t}^{r}$ when other players use $\sigma_{-t}$, we conclude that $u_{t}(x) \geq \delta y_{t}$. Thus $x \in B_{t}$. Since $t$ is arbitrary, $x \in B$.

We prove that the set $A$ is non-empty. As before, let $N_{a}=\left\{t \in N \mid x_{t} \in A\right\}$ and let $N_{r}$ be a complement of $N_{a}$. We show that $\mu\left(N_{a}\right)>0$. If $\mu\left(N_{a}\right)=0$, then $y_{\bullet}$ is identically zero. In this case Int $X \subset C \subset A$. Thus each alternative in the interior of $X$ is unanimously accepted. Take $t \in N_{r}$ and $x \in \operatorname{Int} X$. Then the strategy $\sigma_{t}^{\prime}=\left(x, A_{t}\right)$ improves the payoff of player $t$ at each node where $t$ has to make a proposal, contradicting the assumption that $\sigma$ is a subgame perfect equilibrium.

We prove that the set $B$ is not a singleton. Suppose $B$ is a singleton consisting of point $\bar{x}$ alone. Then $A$ consists of $\bar{x}$ alone as well and $x_{t}=\bar{x}$ for all $t \in N_{a}$. Then and $y_{t}=\lambda u_{t}(\bar{x})$, where $\lambda=\mu\left(N_{a}\right) /\left[1-\delta \mu\left(N_{r}\right)\right]$. Let $\kappa=\delta \lambda$. Notice that $0 \leq \kappa<1$. The set $B$ can now be written as

$$
B=\cap\left\{x \in X \mid u_{t}(x) \geq \kappa u_{t}(\bar{x})\right\} .
$$

If $\kappa=0$, then $B=X$, and if $0<\kappa<1$, then Proposition 8 applies to show that $B$ contains a non-degenerate interval $I(\bar{x}, \kappa)$. In either case, $B$ is a non-singleton, a contradiction.

Since $B$ is a non-empty convex subset of $X$ and it is not a singleton, it has a non-empty interior. Therefore, the set $C$ is non-empty.

We now show that $N_{a}=N$. Suppose not. Take $t \in N_{r}$ and $x \in C$. Then strategy $\sigma_{t}^{\prime}=\left(x, A_{t}\right)$ improves the payoff to player $t$ at each node where $t$ has to make a proposal, contradicting the subgame perfection hypothesis. Since $N_{a}=N$, the expected utility of player $t$ can be written as

$$
y_{t}=\int u_{t}\left(x_{i}\right) d \mu(i)
$$

Finally, we have to show that the proposal $x_{t}$ of player $t$ maximizes the function $u_{t}$ on $B$. Suppose not. Then there is an $x \in \operatorname{Int} B$ such that $u_{t}(x)>u_{t}\left(x_{t}\right)$. As $\operatorname{Int} B \subset C \subset A$, the point $x$ is unanimously accepted. The strategy $\sigma_{t}^{\prime}=\left(x, A_{t}\right)$ is then a profitable deviation from $\sigma_{t}$ by player $t$ at any node where $t$ has to make a proposal, a contradiction.

\section{References}

[1] Jeffrey S. Banks and John Duggan: A Bargaining Model of Collective Choice. American Political Science Review, 94: 73-88, 2000.

[2] Daniel Cardona and Clara Ponsatí: Bargaining One-Dimensional Social Choices. Journal of Economic Theory, doi: 10.1016/j.jet.2006.12.001, 2007.

[3] Seok-Ju Cho and John Duggan: Uniqueness of Stationary Equilibria in a OneDimensional Model of Bargaining. Journal of Economic Theory, 113: 118-130, 2003.

[4] P. Jean-Jacques Herings and Arkadi Predtetchinski: One-Dimensional Bargaining With Markov Recognition Probabilities. Working paper. 
[5] Toshiji Miyakawa Non-cooperative Foundation of $n$-Person Asymmetric Nash Bargaining Solution. Osaka University of Economics, working paper No. 2006-2. 